\title{
Spectral Analysis for Systems of Atoms and Molecules coupled to the Quantized Radiation Field
}

\author{
Volker Bach *
}

FB Mathematik MA 7-2; TU Berlin; Str. d. 17 Juni 136;

D-10623 Berlin; Germany (bach@math.tu-berlin.de)

Jürg Fröhlich

Inst. f. Theoretische Physik; ETH Hönggerberg;

CH-8093 Zürich; Switzerland (juerg@itp.phys.ethz.ch)

Israel Michael Sigal ${ }^{\dagger}$

Dept. of Math.; Univ. of Toronto;

Toronto, M5S 3G3; Canada (sigal@math.toronto.edu)

November 20, 1998

\begin{abstract}
We consider systems of static nuclei and electrons -atoms and molecules-coupled to the quantized radiation field. The interactions between electrons and the soft modes of the quantized electromagnetic field are described by minimal coupling, $\vec{p} \rightarrow \vec{p}-e \vec{A}(\vec{x})$, where $\vec{A}(\vec{x})$ is the electromagnetic vector potential with an ultraviolet cutoff. If the interactions between the electrons and the quantized radiation field are turned off, the atom or molecule is assumed to have at least one bound state. We prove that, for sufficiently small values of the feinstructure constant $\alpha$, the interacting system has a ground state corresponding to the bottom of its energy spectrum and that the excited states of the atom or molecule above the ground state turn into metastable states
\end{abstract}

${ }^{*}$ Heisenberg Fellow of the DFG, supported by SFB 288 of the DFG, the TMR-Network on "PDE and QM".

${ }^{\dagger}$ Supported by NSERC Grant NA 7901 
whose life-times we estimate. Furthermore the energy spectrum is absolutely continuous, except, perhaps, in a small interval above the ground state energy and around the threshold energies of the atom or molecule.

Keywords: Renormalization Group, Spectrum, Resonances, Fock space, QED. 


\section{Introduction and Survey of Results}

\section{I.1 The Quantum Theory of Photons and Nonrelativi- stic, Bound Electrons}

In this paper we continue our mathematical analysis of the standard model of nonrelativistic, quantum-mechanical matter interacting with the quantized radiation field initiated in $[4,5,6]$. The purpose of the present paper is to refine the techniques developed in $[4,5,6]$ in such a way that they can be applied to the study of physically relevant models.

The physical system we are analyzing consists of a finite number of nuclei, treated as static sources, and a finite number of nonrelativistic electrons, e.g., atoms, ions, or molecules in a Born-Oppenheimer approximation, interacting with the soft modes of the quantized electromagnetic field which is cut-off in the ultraviolet. The Hilbert space of pure state vectors of the system is given by

$$
\mathcal{H}:=\mathcal{H}_{e l} \otimes \mathcal{F},
$$

where $\mathcal{H}_{e l}$ is the Hilbert space of some finite number, $N$, of electrons, and $\mathcal{F}$ is the photon Fock space. Thus, in the Schrödinger (configuration-space) representation, $\mathcal{H}_{e l}$ is given by the subspace of totally antisymmetric wave functions in $L^{2}\left[\left(\mathbb{R}^{3} \times \mathbb{Z}_{2}\right)^{N}\right]$, where $\mathbb{R}^{3}$ is the configuration space of a single electron, and $\mathbb{Z}_{2}$ describes its spin, i.e.,

$$
\mathcal{H}_{e l}:=\mathcal{A}_{N} L^{2}\left[\left(\mathbb{R}^{3} \times \mathbb{Z}_{2}\right)^{N}\right]
$$

with $\mathcal{A}_{N}$ being the orthogonal projection onto the subspace of totally antisymmetric wave functions, as required by the Pauli principle.

The one-photon Hilbert space is given by $L^{2}\left[\mathbb{R}^{3} \times \mathbb{Z}_{2}\right]$, where $\mathbb{R}^{3}$ is the photon momentum space and $\mathbb{Z}_{2}$ describes the two independent transversal polarizations of a photon. (Here and above, the integration measure on $\mathbb{R}^{3}$ is Lebesgue measure.) The photon Fock space is then defined by

$$
\mathcal{F}:=\bigoplus_{n=0}^{\infty} \mathcal{S}_{n} L^{2}\left[\left(\mathbb{R}^{3} \times \mathbb{Z}_{2}\right)^{n}\right],
$$

where $\mathcal{S}_{n}$ is the orthogonal projection onto the subspace of totally symmetric $n$-photon wave functions, in accordance with the fact that photons are bosons. It is convenient to represent the Hilbert space $\mathcal{H}$ as the space of antisymmetric, square-integrable wave functions on the $N$-electron configuration 
space with values in the photon Fock space $\mathcal{F}$, i.e.,

$$
\mathcal{H} \cong \mathcal{A}_{N} L^{2}\left[\left(\mathbb{R}^{3} \times \mathbb{Z}_{2}\right)^{N} ; \mathcal{F}\right]
$$

The dynamics of the system is generated by the Hamiltonian

$$
H_{\alpha}^{\prime}:=\sum_{j=1}^{N}\left[\vec{\sigma}_{j} \cdot\left(-i \vec{\nabla}_{\vec{x}_{j}}-2 \pi^{1 / 2} \alpha^{3 / 2} \vec{A}_{\kappa}\left(\alpha \vec{x}_{j}\right)\right)\right]^{2}+V_{c}(x)+H_{f},
$$

where we use units in which $\hbar=1$ and the electron mass equals $1 / 2$. In (I.5), $\vec{\sigma}_{j}=\left(\sigma_{j}^{x}, \sigma_{j}^{y}, \sigma_{j}^{z}\right)$ denotes the three Pauli matrices associated with the $j^{\text {th }}$ electron, $x_{j}$ is its position (in suitable units of length described below), $\alpha$ is the feinstructure constant, and $\vec{A}(\vec{x})$ denotes the quantized vector potential of the transverse modes of the electromagnetic field in the Coulomb gauge, i.e.,

$$
\vec{A}(\vec{x}):=\sum_{\lambda=1,2} \int \frac{d^{3} k \kappa(|\vec{k}| / K)}{\pi \sqrt{2 \omega(\vec{k})}}\left\{\vec{\varepsilon}_{\lambda}(\vec{k}) \mathrm{e}^{-i \vec{k} \cdot \vec{x}} a_{\lambda}^{*}(\vec{k})+\vec{\varepsilon}_{\lambda}(\vec{k})^{*} \mathrm{e}^{i \vec{k} \cdot \vec{x}} a_{\lambda}(\vec{k})\right\},
$$

where $\kappa$ is an entire function of rapid decrease on the real line, e.g., $\kappa(r):=$ $\exp \left(-r^{4}\right)$, cutting off the vector potential in the ultraviolet domain, $\omega(\vec{k}):=$ $|\vec{k}|$ is the frequency of a photon with wave vector $\vec{k}$, and $\vec{\varepsilon}_{\lambda}(\vec{k}), \lambda=1,2$, are photon polarization vectors satisfying

$$
\vec{\varepsilon}_{\lambda}(\vec{k})^{*} \cdot \vec{\varepsilon}_{\mu}(\vec{k})=\delta_{\lambda \mu}, \quad \vec{k} \cdot \vec{\varepsilon}_{\lambda}(\vec{k})=0, \quad \text { for } \lambda, \mu=1,2 .
$$

Moreover, $a_{\lambda}^{*}(\vec{k}), a_{\lambda}(\vec{k})$ are standard creation- and annihilation operators (see, e.g., [25]) on $\mathcal{F}$ obeying the canonical commutation relations

$$
\left[a_{\lambda}^{\#}\left(\vec{k}_{1}\right), a_{\mu}^{\#}\left(\vec{k}_{2}\right)\right]=0, \quad\left[a_{\lambda}\left(\vec{k}_{1}\right), a_{\mu}^{*}\left(\vec{k}_{2}\right)\right]=\delta_{\lambda \mu} \delta\left(\vec{k}_{1}-\vec{k}_{2}\right),
$$

where $a^{\#}=a$ or $a^{*}$. These objects are densely defined, operator-valued tempered distributions on Fock space $\mathcal{F}$. Fock space $\mathcal{F}$ contains a vector $\Omega$, the vacuum vector, uniquely determined, up to a phase, by the properties that $\|\Omega\|=1$ and $a_{\lambda}(\vec{k}) \Omega=0$, for all $\lambda$ and $\vec{k}$. A dense set of vectors in $\mathcal{F}$ is obtained by applying polynomials in $a_{\lambda}^{*}(\lambda=1,2)$, smeared out with test functions, to the vacuum vector $\Omega$.

The term $V_{c}$ on the right side of Eqn. (I.5) defining the Hamiltonian is the Coulomb potential describing the electrostatic interactions between electrons and nuclei. In our units, it is given by

$$
V_{c}(x):=\sum_{j=1}^{N} \sum_{m=1}^{M} \frac{-Z_{m}}{\left|\vec{x}_{j}-\vec{R}_{m}\right|}+\sum_{1 \leq i<j \leq N} \frac{1}{\left|\vec{x}_{i}-\vec{x}_{j}\right|},
$$


where $x \equiv\left(\vec{x}_{1}, \ldots, \vec{x}_{N}\right) \in \mathbb{R}^{3 N}$, and $\vec{R}_{1}, \ldots, \vec{R}_{M} \in \mathbb{R}^{3}$ are the positions of $M$ static nuclei with atomic numbers $Z_{1}, \ldots, Z_{M}$.

Finally, the Hamiltonian of the free quantized electromagnetic field, $H_{f}$, is given by

$$
H_{f}:=\sum_{\lambda=1,2} \int d^{3} k a_{\lambda}^{*}(\vec{k}) \omega(\vec{k}) a_{\lambda}(\vec{k})
$$

The r.s. of Eqn. (I.10) defines a densely defined, positive, self-adjoint operator on $\mathcal{F}$ with absolutely continuous spectrum on the positive half-axis and a simple eigenvalue at 0 corresponding to the eigenvector $\Omega$.

Next, we comment on the units chosen in (I.5). Length (and hence the positions, $\vec{x}_{j}$, of the electrons and, $\vec{R}_{m}$, of the nuclei) is measured in units of half a Bohr radius, $\frac{1}{2} r_{\text {Bohr }}=\frac{\hbar^{2}}{2 m_{e l} e^{2}}$, expressed in Gaussian units, where $m_{e l}$ is the mass of an electron and $-e$ its electric charge. Photon wave lengths are measured in units of $\alpha$ times half a Bohr radius, $\frac{\alpha}{2} r_{\text {Bohr }}$, i.e., the unit for photon wave vectors is $2 \alpha^{-1} r_{\mathrm{Bohr}}{ }^{-1}$. The energy unit is chosen to be 4 Rydberg, with $4 R y=\frac{2 e^{2}}{r_{\text {Bohr }}}$. The ultraviolet cutoff of the radiation field imposed by the function $\kappa(|k| / K)$ used in the definition of the vector potential $\vec{A}(\vec{x})$ turns off interactions between electrons and photons with energy large compared to $K \cdot 4 R y$. The physical value of the feinstructure constant $\alpha$ is $\approx 1 / 137$. In this paper, $\alpha$ plays the rôle of a small, dimensionless number. Our results hold for sufficiently small values of $\alpha$. We shall not verify that the radii of convergence in $\alpha$ of our analytical constructions cover the physical point $\alpha=\alpha_{(\mathrm{phys})} \approx 1 / 137$ (such a verification would presumably require numerical work on a computer).

Our main concern in this paper is to analyze properties of the energy spectrum of the Hamiltonian $H_{\alpha}^{\prime}$ and to study resonances for the dynamics generated by $H_{\alpha}^{\prime}$ and estimate their life times. We shall show that, for sufficiently small values of $\alpha>0, H_{\alpha}^{\prime}$ has a normalizable ground state corresponding to the minimum of its spectrum and that "most" of its spectrum is absolutely continuous. Furthermore, we shall show that the excited bound states of the atom or ion (for purely technical reason, we exclude molecules here) with electrons decoupled from the radiation field, i.e., the bound states of $H_{0}$, turn into metastable states of finite life time when that coupling is turned on. We show that Fermi's golden rule [24] yields an accurate estimate of the life times of metastable states, and we provide a rigorous justification of Bethe's formula [10, 9] for the Lamb shift.

In order to describe our results and methods more precisely, we start with a discussion of the spectral properties of the unperturbed Hamiltonian 
(electrons decoupled from the radiation field),

$$
H_{0} \equiv H_{\alpha=0}^{\prime}=H_{e l}+H_{f}
$$

where $H_{e l}$ is the usual atomic (or molecular) Hamiltonian defined by

$$
H_{e l}:=\sum_{j=1}^{N}-\Delta_{\vec{x}_{j}}+V_{c}(x) .
$$

We recall some key properties of $H_{e l}$, viewed as an operator on $\mathcal{H}_{e l}$. For details and proofs, see [24, 13] and references given there. We first note that the potential $V_{c}$ is a perturbation of the kinetic energy operator $\Delta_{x}:=$ $\sum_{j=1}^{N} \Delta_{\vec{x}_{j}}$ with zero relative bound. Thus $H_{e l}$ is a semibounded, self-adjoint operator on the domain $\mathcal{D}\left(H_{e l}\right)=\mathcal{D}\left(\Delta_{x}\right)=\mathcal{H}_{e l} \cap H^{2}\left[\left(\mathbb{R}^{3} \times \mathbb{Z}_{2}\right)^{N}\right]$, where $H^{2}$ is the usual Sobolev space. The essential spectrum of $H_{e l}$ is given by $\sigma_{\text {ess }}\left(H_{e l}\right)=[\Sigma, \infty)$, where $\Sigma$ is the infimum of the spectrum of $H_{e l}$, with $N$ replaced by $N-1$, as follows from the HVZ theorem (see, e.g., [13]). Thus, $\Sigma$ is the ionization threshold.

In what follows, we shall make the following assumption about the atom, ion, or molecule under consideration, represented by the parameters $N$, $\vec{R}_{1}, \ldots, \vec{R}_{M}$, and $Z_{1}, \ldots, Z_{M}$. For the proof of existence of a ground state of $H_{\alpha}^{\prime}$ in Section II, we assume that $H_{e l}$ has at least one eigenvalue $E_{0}$ below the ionization threshold $\Sigma$, i.e., we require that

$$
E_{0}:=\inf \sigma\left(H_{e l}\right)<\Sigma:=\inf \sigma_{\mathrm{ess}}\left(H_{e l}\right) .
$$

The contents of Section III on the life times of metastable states is nontrivial only if, besides $E_{0}, H_{e l}$ has at least one further eigenvalue $E_{1}$, with $E_{0}<E_{1}<\Sigma$, i.e., in Section III we additionally require that the spectrum

$$
\sigma\left(H_{e l}\right)=\left\{E_{0}, E_{1}, \ldots\right\} \cup[\Sigma, \infty)
$$

of $H_{e l}$ consists of eigenvalues $E_{0}<E_{1}<E_{2}<\cdots \leq \Sigma$, of finite multiplicity below $\Sigma$, possibly with an accumulation point at $\Sigma$ and (absolutely) continuous spectrum in $[\Sigma, \infty)$; see Fig. 1. In Section III we shall also require an assumption saying, roughly speaking, that there are no accidental selection rules in the system described by $H_{e l}$, which could prevent excited eigenstates of $H_{e l}$ from decaying radiatively (in $2^{\text {nd }}$ order in the relevant coupling constant).

For positive ions and (neutral) atoms or molecules our assumption (I.14) is justified, as it is known that if $N \leq \sum_{m=1}^{M} Z_{m}$ then $H_{e l}$ has infinitely many eigenvalues of finite multiplicity below $\Sigma$ [31]. In the case of negatively 


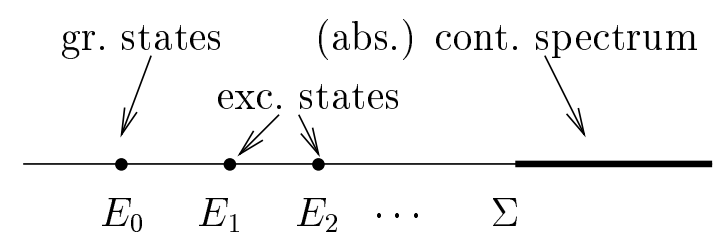

Figure 1: The Spectrum of $H_{e l}$

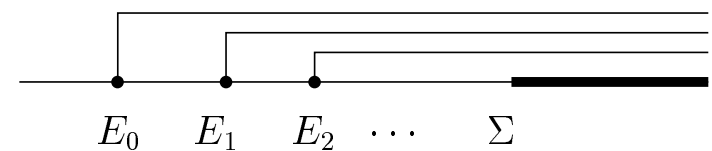

Figure 2: The Spectrum of $H_{0}=H_{e l} \otimes \mathbf{1}+\mathbf{1} \otimes H_{f}$

ionized atoms or molecules, i.e., if $N>\sum_{m=1}^{M} Z_{m}$, the question, whether $H_{e l}$ has isolated eigenvalues below the ionization treshold, is more subtle. Indeed, if $N \geq \sum_{m=1}^{M}\left(2 Z_{m}+1\right)$ then $H_{e l}$ has no eigenvalues at all [23] (see [27, 28] for earlier results).

Assuming that the atomic numbers of the nuclei are such that $E_{0}<\Sigma$, i.e., that $H_{e l}$ has isolated eigenvalues below the ionization threshold, it tends to be an open question whether the ground state corresponding to the energy $E_{0}$ is unique or not, except when $N=1$ or $=2$, in which case a standard Perron-Frobenius argument proves uniqueness. (Non-uniqueness for $N \geq 3$ may arise as a consequence of the Pauli principle.)

The spectrum of the photon Hamiltonian $H_{f}$ consists of a simple eigenvalue at 0 , corresponding to the vacuum vector $\Omega \in \mathcal{F}$, and absolutely continuous spectrum (of infinite multiplicity) covering the half-axis $[0, \infty$ ). Consequently, by separation of variables, the unperturbed Hamiltonian $H_{0}=$ $H_{e l}+H_{f}$ on $\mathcal{F}$ has spectrum

$$
\sigma\left(H_{0}\right)=\overline{\sigma\left(H_{e l}\right)+\sigma\left(H_{f}\right)} .
$$

The point spectrum of $H_{0}$ is the same as the point spectrum of $H_{e l}$, i.e., it consists of the eigenvalues $\left\{E_{j}\right\}_{j=0,1,2, \ldots}$ (corresponding to the eigenvectors $\varphi_{j, \ell} \otimes \Omega$, where $\left\{\varphi_{j, \ell}\right\}_{\ell=1,2, \ldots, n_{j}}$ is an orthonormal basis of eigenvectors of $H_{e l}$ corresponding to the eigenvalue $E_{j}$ of multiplicity $n_{j}$ ). The continuous spectrum of $H_{0}$ covers the half-axis $\left[E_{0}, \infty\right)$ and consists of a union of branches $\left[E_{j}, \infty\right)$ starting at the eigenvalues $E_{j}$ and the branch $[\Sigma, \infty)$; as indicated in Fig. 2.

We note that the ground state energy $E_{0}=\inf \sigma\left(H_{e l}\right)$ of the atom or molecule in the absence of the quantized radiation field coincides with the ground state energy $E_{0} \equiv E_{0}(\alpha=0)=\inf \sigma\left(H_{0}\right)$ of the system of an atom 
or molecule in the presence of photons, but decoupled from them. But, while $E_{0}$ is an isolated eigenvalue of $H_{e l}$, it lies at the tip of a branch of continuous spectrum of the Hamiltonian $H_{0}$. Similarly, the energies $E_{1}, E_{2}, \ldots$ are isolated eigenvalues of $H_{e l}$; but they are eigenvalues of $H_{0}$ imbedded in continuous spectrum of $H_{0}$, and each $E_{j}$ is the threshold of a branch of continuous spectrum of $H_{0}$.

These spectral properties of $H_{0}$ make it a difficult problem to analyze, mathematically rigorously, the fate of the eigenvalues $E_{j}$ of $H_{0}$, and the nature of the energy spectrum of the interacting system described by the Hamiltonian $H_{\alpha}^{\prime}$ introduced in (I.5), for $\alpha>0$. Although the perturbation, $W_{\alpha}^{\prime}:=H_{\alpha}^{\prime}-H_{0}$, is a small perturbation of $H_{0}$, general analytical methods to deal with this type of problem in perturbation theory do not appear to be available. In $[5,6]$, we have started to develop such methods, tailor-made to analyze a class of Hamiltonians describing interactions between nonrelativistic quantum-mechanical matter and the radiation field. In this paper we extend those methods to the Hamiltonian $H_{\alpha}^{\prime}\left(=H_{0}+W_{\alpha}^{\prime}\right)$ of Eqn. (I.5), which describes much of the physics of light atoms or molecules interacting with the quantized electromagnetic field (within the Born-Oppenheimer approximation). For background material concerning the physics described by $H_{\alpha}^{\prime}$, see e.g., $[11,12]$ and references given there.

\section{I.2 Survey of Main Results}

In the next section, I.3, we consider the structure and properties of the perturbation $W_{\alpha}^{\prime}$ in the Hamiltonian $H_{\alpha}^{\prime}=H_{0}+W_{\alpha}^{\prime}$ of (I.5). The strength of the perturbation $W_{\alpha}^{\prime}$ relative to $H_{0}$ is measured by the parameter

$$
g:=(\alpha K)^{3 / 2}
$$

where $K$ is the ultraviolet cutoff in the electromagnetic vector potential $\vec{A}$, as described in Eqn. (I.6). The parameter $K$ is a "dimensionless energy scale" given by the photon energy above which interactions between electrons and the radiation field are cut off, divided by 4 Ry. In Section I.3 we prove that if $g$ is sufficiently small then the interaction $W_{g}$ is bounded by $H_{0}$, in the sense of Kato [22, 25], with relative bound strictly smaller than 1 . This proves that, for small $\alpha, H_{\alpha}^{\prime}$ is bounded from below and self-adjoint on the domain of $H_{0}$; see Corollary I.2. (Under somewhat weaker assumptions one can prove that $H_{\alpha}^{\prime}$ defines a semibounded quadratic form on an appropriate core. It is not easy, however, to characterize the domain of the corresponding selfadjoint operator.) Since $g:=(\alpha K)^{3 / 2}$ is the relevant coupling parameter, we 
henceforth write

$$
H_{g}:=H_{\alpha=g^{2 / 3} / K}^{\prime}, \quad W_{g}:=W_{\alpha=g^{2 / 3} / K}^{\prime} .
$$

We should emphasize that our bounds on $g=(\alpha K)^{3 / 2}$ become poor, as the number of electrons, $N$, becomes large. This does not mean that the Hamiltonian $H_{g}$ is ill-defined or unbounded from below, for large values of $\alpha$, as long as the ultraviolet cutoff $K$ is kept finite. In fact, using pathspace methods one can rigorously construct the semi-group $\exp \left(-t H_{g}\right)$, for $t \geq 0$, and prove that it is self-adjoint, strongly continuous in $t>0$ with $\exp \left(-t H_{g}\right) \rightarrow \mathbf{1}$, as $t \rightarrow 0$, for arbitrary values of $\alpha$ and $N$, as long as $K<\infty$; (see $[17,30]$ for various ingredients of the proof). However, for the purposes of the analysis presented in Section III, Kato- (or quadratic-form-) boundedness of $W_{g}$ in terms of $H_{0}$ is an important property.

In Section II we address the question whether $H_{g}$ has a ground state corresponding to an eigenvalue $E_{0}(g):=\inf \sigma\left(H_{g}\right)$. Our main result is Theorem II.1, which answers this question in the affirmative, as long as the effective coupling constant $g$ is sufficiently small.

The method used in Section II is "non-perturbative" but non-constructive. For this reason, they do not enable us to estimate the multiplicity of the ground state energy $E_{0}(g)$. However, if the number $N$ of electrons is $=1$ or $=2$, and if the Zeeman terms in the Hamiltonian $H_{g}$ are set to 0 , then we can construct an $L^{2}$-space representation of the photon Fock space $\mathcal{F}$ ("electric field" representation) with the property that $\exp \left(-t H_{g}\right)$ is positivitypreserving on $\mathcal{H}_{e l} \otimes \mathcal{F}$, where $\mathcal{H}_{e l}$ is taken in the usual Schrödinger configuration-space representation, and, for $N=2$, only spin-singlet states are considered. Modulo ergodicity of $\exp \left(-t H_{g}\right)$ (see [24]), which we have not checked, a Perron-Frobenius argument then establishes uniqueness of the groundstate.

It is worthwhile to point out some (fairly standard, but) fundamental consequences of the existence of a ground state of $H_{g}$ for scattering theory: Using straightforward variants of methods developed in [18, 19, 20, 2, 3], one can construct Möller (wave) operators, $\Omega_{ \pm}$, such that the range of $\Omega_{+}\left(\Omega_{-}\right)$ describes asymptotic states of the system consisting of an atom (or molecule) in a ground state accompanied by an outgoing cloud of freely moving photons. The obvious conjecture is that $\operatorname{Ran}\left\{\Omega_{+}\right\}=\operatorname{Ran}\left\{\Omega_{-}\right\}=: \mathcal{H}_{\text {asy }}$, where $\mathcal{H}_{\text {asy }}$ is isomorphic to $\mathcal{H}_{g}^{(0)} \otimes \mathcal{F}$, and where $\mathcal{H}_{g}^{(0)}$ is the space of ground states of $H_{g}$. This conjecture, called "asymptotic completeness", would imply the unitarity of the scattering matrix for the scattering of photons off an atom or molecule below the ionization threshold. We are miles away from proving this conjecture! But, in a drastically simpler model of massive photons and confined electrons, it has recently been proven in $[16,14]$. 
In Section III, we prove that, outside small neighbourhoods of $E_{0}$ and $\Sigma$ and below $\Sigma$, the spectrum of $H_{g}$ is purely absolutely continuous (Outside small neighbourhoods of the thresholds of $H_{e l}$ and above $\Sigma$, the spectrum of $H_{g}$ can be shown to be purely absolutely continuous by using the methods in $[5,7]$. In particular, $H_{g}$ does not have any eigenvalues in the vicinity of the imbedded eigenvalues $E_{1}<E_{2}<\cdots<E_{n}<\Sigma-\delta$, of $H_{0}$, where $\delta$ is a small positive constant depending on $g$ and $n$. We will, however, make the idea rather precise that imbedded eigenvalues of $H_{0}$ give rise to metastable states of $H_{g}$, and we shall estimate the life time, $\propto g^{2}$, of these metastable states up to an error term $\mathcal{O}\left(g^{2+\varepsilon}\right)$, for some $\varepsilon>0$.

Our notion of resonance is based on dilatation analyticity. In order to state our ideas simply, we assume that there is only one atomic nucleus ( $M=1$ in Eqn. (I.9)) of atomic number $Z=Z_{1} \leq 2 N$ located at $\vec{R}_{1}=0$; (the general case of an arbitrary, finite number $M$ of nuclei can be studied, too, by borrowing ideas developed in [21]). To describe dilatation analyticity, we start by scaling the positions, $\vec{x}_{j}$, of the electrons and the momenta, $\vec{k}$, of the photons by

$$
\vec{x}_{j} \mapsto e^{\theta} \vec{x}_{j}, \quad \vec{k} \mapsto e^{-\theta} \vec{k}
$$

When $\theta$ is real, the transformations (I.18) determine a unitary transformation $U_{\theta}$ on the Hilbert space $\mathcal{H}$ defined in Eqns. (I.1)-(I.4). It is easy to see that the subspace, $\mathcal{D} \subseteq \mathcal{H}$, of vectors, $\psi$, with the property that $\psi(\theta):=U_{\theta} \psi$ is analytic in $\theta$, for $|\operatorname{Im} \theta|<\pi / 2$, is dense in $\mathcal{H}$. Furthermore, one easily checks that

$$
U_{\theta} H_{f} U_{\theta}^{*}=e^{-\theta} H_{f} .
$$

These facts, combined with well-known results $[1,8]$ on dilatation analyticity for Schrödinger operators, show that, for arbitrary $\psi, \varphi \in \mathcal{D}$, the function

$$
F_{\psi, \varphi}^{0}(\theta, z):=\left\langle\psi_{\bar{\theta}} \mid\left(z-H_{0}(\theta)\right)^{-1} \varphi(\theta)\right\rangle,
$$

where $H_{0}(\theta)=U_{\theta} H_{0} U_{\theta}^{-1}$, is independent of $\theta$, for $|\operatorname{Im} \theta|<\pi / 2$, and, for $\operatorname{Im} \theta=: \vartheta$ fixed, $F_{\psi, \varphi}^{0}(\theta, z)$ is analytic in $z$ in the complement of the shaded region depicted in Fig. 3

In Section III, we construct the function

$$
F_{\psi, \varphi}^{g}(\theta, z):=\left\langle\psi_{\bar{\theta}} \mid\left(z-H_{g}(\theta)\right)^{-1} \varphi(\theta)\right\rangle,
$$

where $H_{g}(\theta)=U_{\theta} H_{g} U_{\theta}^{-1}$, with $H_{g}$ as in (I.5), and we show that, for our choice of an ultraviolet cutoff $\kappa$ (see (I.6) and below), and for arbitrary $\psi$ 


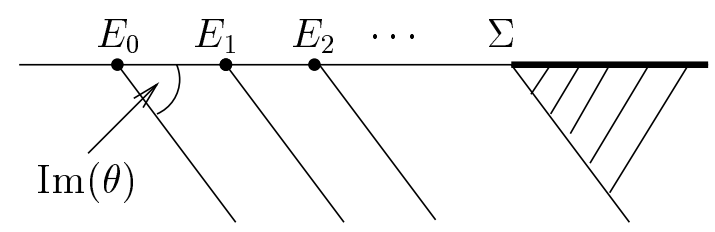

Figure 3: A projection of the Riemann surface of $z \mapsto F_{\psi, \varphi}^{0}(\theta, z)$ onto the energy plane

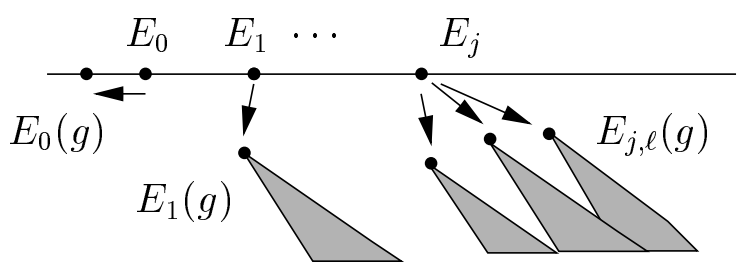

Figure 4: A projection of the Riemann surface of $z \mapsto F_{\psi, \varphi}^{g}(\theta, z)$ onto the energy plane

and $\varphi$ in $\mathcal{D}, F_{\psi, \varphi}^{g}(\theta, z)$ is independent of $\theta$, for $|\operatorname{Im} \theta|<\pi / 4$ small enough, and, for $\operatorname{Im} \theta=: \vartheta$ fixed, $F_{\psi, \varphi}^{g}(\theta, z)$ is analytic in $z$ in the complement of the shaded region depicted in Fig. 4 . It thus provides an analytic continuation of the matrix element $F_{\psi, \varphi}^{g}(0, z)$ of the resolvent of $H_{g}-z$ in $z$ from the upper half plane into the lower half plane outside the spectrum of $H_{g}(\theta)$. This implies the absolute continuity of the spectrum of $H_{g}$ for those energies which are contained in the resolvent set of $H_{g}(\theta)$ (see Corollary III.2).

In Section III, Eqns. (III.7)-(III.10), we introduce a notion of resonance energy $E_{j}(g)$ corresponding to the energy $E_{j}$ of the $j^{\text {th }}$ excited state of the atom or molecule. We show that the Lamb shift, $\operatorname{Re}\left\{E_{j}(g)-E_{j}\right\}$, is given by Bethe's formula and $\operatorname{Im}\left\{E_{j}(g)\right\}$ is given by Fermi's Golden Rule, to order $g^{2}$, with error terms that we prove to be $\mathcal{O}\left(g^{2+\varepsilon}\right)$, for any $0<\varepsilon<1 / 3$. If there are no miraculous selection rules forbidding the decay of the $j^{\text {th }}$ excited state of the atom to first order in $g$ then

$$
\operatorname{Im}\left\{E_{j}(g)\right\}=\Gamma_{j} g^{2}+\mathcal{O}\left(g^{2+\varepsilon}\right),
$$

with $\Gamma_{j}$ strictly positive.

It then follows by standard reasoning that, for $g>0$ sufficiently small, the spectrum of $H_{g}$ is purely absolutely continuous in a neighbourhood of every eigenvalue $E_{j}, j \geq 1$, of $H_{e l}$ for which $\Gamma_{j}>0$.

What we are really looking after, from a physics point of view, is a precise understanding of the decay of the excited states of the atom under the time evolution $\exp \left[-i t H_{g}\right]$, as $t$ becomes large. Let $\delta:=\operatorname{dist}\left(E_{j}, \sigma\left(H_{e l}\right) \backslash\left\{E_{j}\right\}\right)>$ 
0 , set $\mathcal{I}_{j}(\delta / 2):=\left(E_{j}-\delta / 2, E_{j}+\delta / 2\right)$, and notice that $\mathcal{I}_{j}(\delta / 2)$ is an open interval containing $E_{j}$ and such that $\operatorname{dist}\left(\mathcal{I}_{j}, \sigma\left(H_{e l}\right) \backslash\left\{E_{j}\right\}\right)=\delta / 2$. Let $F_{j}$ denote a smooth characteristic function of $\mathcal{I}_{j}(\delta / 2)$ (see Subsection III.1). We shall identify a " $j^{\text {th }}$ excited state" of the atom with a vector of the form

$$
\psi_{j}:=F_{j}^{1 / 2}\left(H_{g}\right) P_{e l, j} \exp \left[-g^{-2} H_{f}\right] \eta
$$

(see (III.14)), where $P_{e l, j}$ is the projection onto the eigenspace of $H_{e l}$ corresponding to the eigenvalue $E_{j}$, and the operator $\exp \left[-g^{-2} H_{f}\right]$ essentially eliminates high-energy photons of energy larger than $g^{2}$ in the state $\eta$, which is assumed to be dilatation analytic. An example for a state $\eta$ is given by

$$
\eta=\varphi_{j, \ell} \otimes \Omega
$$

where $\varphi_{j, \ell}$ is an eigenvector of $H_{e l}$ with corresponding to the eigenvalue $E_{j}$.

We then show that, for any $0<\varepsilon<1 / 3$, there exists a constant $C \geq 0$ and, for any $N \in \mathbb{N}$, a constant $C_{N} \geq 0$ such that

$$
\begin{aligned}
& \left|\left\langle\psi_{j} \mid \exp \left[-i t H_{g}\right] \psi_{j}\right\rangle\right| \\
& \quad \leq B_{\eta}^{2}\left(C \ln (1 / g) \exp \left[-t\left(g^{2} \Gamma_{j}-C g^{2+\varepsilon}\right)\right]+C_{N} t^{-N} g^{4}\right),
\end{aligned}
$$

where $B_{\eta}:=1+\sup _{|\theta| \leq \theta_{0}}\|\eta(\theta)\|$. This estimate implies that, given $\varepsilon>0$, there is a finite constant $C_{\varepsilon}>0$ such that, for $t>C_{\varepsilon} \Gamma_{j}^{-1} g^{-2} \ln \ln (1 / g)$,

$$
\left|\left\langle\psi_{j} \mid e^{-i t H_{g}} \psi_{j}\right\rangle\right| \leq \varepsilon \text {. }
$$

We remark that it is known from other methods (see e.g. [15]) that, given $\varepsilon>0$, there is a constant $D_{\varepsilon}>0$ such that, for $0 \leq t<D_{\varepsilon} g^{-2}$,

$$
\left|\left\langle\psi_{j} \mid e^{-i t H_{g}} \psi_{j}\right\rangle\right| \geq \varepsilon \text {. }
$$

Estimate (I.26) shows that the state $\psi_{j}$ decays, with a life-time bounded above by $\mathcal{O}\left(g^{-2} \ln \ln (1 / g)\right)$. It is a typical example for the kind of estimates we are able to prove with the help of the methods developed in Section III.

\section{I.3 Relative Bounds, Self-Adjointness, and Dilatation Analyticity}

We return to Eqn. (I.5), which we write as

$$
H_{g}=H_{0}+W_{g},
$$


where $H_{0}$ is defined in (I.11), and we obtain

$$
\begin{aligned}
W_{g}=\sum_{j=1}^{N}\{- & 4 \pi^{1 / 2} \alpha^{3 / 2} \vec{A}_{\kappa}\left(\alpha \vec{x}_{j}\right) \cdot\left(-i \vec{\nabla}_{\vec{x}_{j}}\right)+2 \pi \alpha^{3} \vec{A}_{\kappa}^{2}\left(\alpha \vec{x}_{j}\right) \\
+ & \left.2 \pi^{1 / 2} \alpha^{5 / 2} \vec{\sigma}_{j} \cdot\left(\vec{\nabla} \times \vec{A}_{\kappa}\right)\left(\alpha \vec{x}_{j}\right)\right\}\left.\right|_{\alpha=g^{2 / 3} / K}
\end{aligned}
$$

from expanding the square in (I.5). Our first goal in this subsection is to prove in Corollary I.2 that $W_{g}$ is defined on $\mathcal{D}\left(H_{0}\right)$ and obeys the bound

$$
\left\|W_{g}\left|H_{0}+i C(N)\right|^{-1}\right\| \leq C^{\prime}(N)(\alpha K)^{3 / 2},
$$

for some constants $C(N), C^{\prime}(N) \geq 0$. This establishes the semiboundedness and self-adointness of $H_{g}$ on $\mathcal{D}\left(H_{0}\right)$ for $g:=(\alpha K)^{3 / 2}<C^{\prime}(N)^{-1}$. Our second goal is to establish the dilatation analyticity of $W_{g}(\theta):=U_{\theta} W_{g} U_{\theta}^{-1}$ (see Corollary I.3), where $U_{\theta}$ is the dilatation operator defined in (I.18), i.e., we prove that $\theta \mapsto W_{g}(\theta)$ is an analytic function on $D\left(0, \theta_{0}\right):=\left\{z \in \mathbb{C}|| z \mid<\theta_{0}\right\}$ with values in $\mathcal{B}\left(\mathcal{D}\left(H_{0}\right) ; \mathcal{H}\right)$, the bounded operators from $\mathcal{D}\left(H_{0}\right)$ to $\mathcal{H}$, for some $\theta_{0}>0$. We establish this property by observing that the coupling functions in $W_{g}(\theta)$ are analytic in $\theta$, pointwise in the other parameters, and by verifying a bound similar to (I.30), namely,

$$
\left\|W_{g}(\theta)\left|H_{0}+i C(N, \theta)\right|^{-1}\right\| \leq C^{\prime}(N, \theta) g,
$$

for some constants $C(N, \theta), C^{\prime}(N, \theta) \geq 0$. In fact, Eqn. (I.30) is just the special case $\theta=0$ in Eqn. (I.31). Finally, we establish the dilatation analyticity of $H_{g}(\theta):=U_{\theta} H_{g} U_{\theta}^{-1}$ in Corollary I.4, assuming that $H_{e l}=-\Delta_{x}+V_{c}(x)$ is the Hamiltonian of an atom, i.e., $M=1$. This simplifying assumption could be avoided by using exterior dilatations [21, 29], at the expense of having to deal with more involved estimates; we do not carry out this analysis here.

We characterize the coupling functions in $W_{g}(\theta)$ in terms of the following functions,

$$
\vec{G}_{\vec{x}}(\vec{k}, \lambda ; \theta):=\frac{\sqrt{2} e^{-\theta / 2} \kappa\left(e^{-\theta}|\vec{k}| / K\right)}{\sqrt{\pi K^{3} \omega(\vec{k})}} \exp [-i \alpha \vec{k} \cdot \vec{x}] \vec{\varepsilon}_{\lambda}(\vec{k})
$$

and

$$
\vec{B}_{\vec{x}}(\vec{k}, \lambda ; \theta):=\frac{\alpha \sqrt{2} e^{-3 \theta / 2} \kappa\left(e^{-\theta}|\vec{k}| / K\right)}{i \sqrt{\pi K^{3} \omega(\vec{k})}} \exp [-i \alpha \vec{k} \cdot \vec{x}]\left(\vec{k} \times \vec{\varepsilon}_{\lambda}(\vec{k})\right),
$$


where we introduce a dilatation parameter $\theta \in D\left(0, \theta_{0}\right) \subseteq \mathbb{C}$, for some $\theta_{0}>0$ sufficiently small. Note that, pointwise for every $\vec{x}, \vec{k} \in \mathbb{R}^{3}$ and $\lambda \in \mathbb{Z}_{2}$, the maps $\theta \mapsto \vec{G}_{\vec{x}}(\vec{k}, \lambda ; \theta)$ and $\theta \mapsto \vec{B}_{\vec{x}}(\vec{k}, \lambda ; \theta)$ are analytic in $D\left(0, \theta_{0}\right)$. Furthermore, we notice that $\vec{B}_{\vec{x}}(\vec{k}, \lambda ; 0)=\vec{\nabla} \times \vec{G}_{\vec{x}}(\vec{k}, \lambda ; 0)$. By means of $\vec{G}_{\vec{x}}(\vec{k}, \lambda ; \theta)$ and $\vec{B}_{\vec{x}}(\vec{k}, \lambda ; \theta)$ we define the following functions on $\mathbb{R}^{3} \times \mathbb{Z}_{2}$ with values in the operators on $\mathcal{H}_{e l}$,

$$
\begin{gathered}
w_{1,0}(\vec{k}, \lambda ; \theta):=w_{0,1}(\vec{k}, \lambda ; \bar{\theta})^{*}:=\sum_{j=1}^{N}\left\{-2 \vec{G}_{\vec{x}_{j}}(\vec{k}, \lambda ; \theta) \cdot \vec{p}_{j}+\vec{\sigma}_{j} \cdot \vec{B}_{\vec{x}_{j}}(\vec{k}, \lambda ; \theta)\right\}, \\
w_{2,0}\left(\vec{k}_{1}, \lambda_{1} ; \vec{k}_{2}, \lambda_{2} ; \theta\right):=w_{0,2}\left(\vec{k}_{1}, \lambda_{1} ; \vec{k}_{2}, \lambda_{2} ; \bar{\theta}\right)^{*} \\
:=\sum_{j=1}^{N}\left\{\vec{G}_{\vec{x}_{j}}\left(\vec{k}_{1}, \lambda_{1} ; \theta\right) \cdot \vec{G}_{\vec{x}_{j}}\left(\vec{k}_{2}, \lambda_{2} ; \theta\right)\right\} \\
w_{1,1}\left(\vec{k}_{1}, \lambda_{1} ; \vec{k}_{2}, \lambda_{2} ; \theta\right):=\sum_{j=1}^{N}\left\{\vec{G}_{\vec{x}_{j}}\left(\vec{k}_{1}, \lambda_{1} ; \bar{\theta}\right)^{*} \cdot \vec{G}_{\vec{x}_{j}}\left(\vec{k}_{2}, \lambda_{2} ; \theta\right)\right. \\
\left.\quad+\vec{G}_{\vec{x}_{j}}\left(\vec{k}_{1}, \lambda_{1} ; \theta\right) \cdot \vec{G}_{\vec{x}_{j}}\left(\vec{k}_{2}, \lambda_{2} ; \bar{\theta}\right)^{*}\right\}
\end{gathered}
$$

and these, in turn, serve as coupling functions for the operators defined by

$$
\begin{array}{r}
W_{m, n}(\theta):=\sum_{\lambda_{1}, \ldots, \lambda_{m+n}=1,2} \int d^{3} k_{1} \cdots d^{3} k_{m+n} w_{m, n}\left(\vec{k}_{1}, \lambda_{1} ; \ldots, \vec{k}_{m+n}, \lambda_{m+n} ; \theta\right) \\
a_{\lambda_{1}}^{*}\left(\vec{k}_{1}\right) \cdots a_{\lambda_{m}}^{*}\left(\vec{k}_{m}\right) a_{\lambda_{m+1}}\left(\vec{k}_{m+1}\right) \cdots a_{\lambda_{m+n}}\left(\vec{k}_{m+n}\right) .(\mathrm{I} .37)
\end{array}
$$

Then we observe that, after normal ordering, the (dilated) interaction $W_{g}(\theta)$ reads

$$
W_{g}(\theta)=\sum_{m+n \leq 2} g^{m+n} W_{m n}(\theta)+g^{2} C_{n o}
$$

where $C_{n o}$ is defined by $C_{n o}:=2 N \int\left|\vec{G}_{\vec{x}}(\vec{k}, 1 ; 0)\right|^{2} d^{3} k$, which is independent of $x$, and $g:=(\alpha K)^{3 / 2}$. Henceforth and consistent with our previous definitions, we omit $\theta$ in our notation in the undilated case, $\theta=0$, writing

$$
\begin{aligned}
\vec{G}_{\vec{x}}(\vec{k}, \lambda):=\vec{G}_{\vec{x}}(\vec{k}, \lambda ; 0) & , \quad \vec{B}_{\vec{x}}(\vec{k}, \lambda):=\vec{B}_{\vec{x}}(\vec{k}, \lambda ; 0) \\
w_{m, n}\left(\vec{k}_{1}, \lambda_{1} ; \ldots, \vec{k}_{m+n}, \lambda_{m+n}\right) & :=w_{m, n}\left(\vec{k}_{1}, \lambda_{1} ; \ldots, \vec{k}_{m+n}, \lambda_{m+n} ; 0\right) \\
W_{m, n} & :=W_{m, n}(0)
\end{aligned}
$$


Next, we define $J: \mathbb{R}^{3} \rightarrow \mathbb{R}_{+}$to be the smallest function such that

$$
\left\|w_{1,0}(\vec{k}, \lambda ; \theta)\left(-\Delta_{x}+1\right)^{-1 / 2}\right\|, \quad\left\|w_{0,1}(\vec{k}, \lambda ; \theta)\left(-\Delta_{x}+1\right)^{-1 / 2}\right\| \leq J(\vec{k})
$$

holds, for all $|\theta| \leq \theta_{0}$ and $(\vec{k}, \lambda) \in \mathbb{R}^{3} \times \mathbb{Z}_{2}$, and such that

$$
\begin{aligned}
\left\|w_{2,0}\left(\vec{k}_{1}, \lambda_{1} ; \vec{k}_{2}, \lambda_{2} ; \theta\right)\right\|, \quad & \left\|w_{1,1}\left(\vec{k}_{1}, \lambda_{1} ; \vec{k}_{2}, \lambda_{2} ; \theta\right)\right\|, \\
& \left\|w_{0,2}\left(\vec{k}_{1}, \lambda_{1} ; \vec{k}_{2}, \lambda_{2} ; \theta\right)\right\| \leq J\left(\vec{k}_{1}\right) J\left(\vec{k}_{2}\right)
\end{aligned}
$$

holds, for all $|\theta| \leq \theta_{0}$ and $\left(\vec{k}_{1}, \lambda_{1}\right),\left(\vec{k}_{2}, \lambda_{2}\right) \in \mathbb{R}^{3} \times \mathbb{Z}_{2}$. Note that, due to $\pm i \vec{\nabla}_{\vec{x}_{j}} \leq\left(-\Delta_{\vec{x}}+1\right)^{1 / 2}$, we have that

$$
J(\vec{k}) \leq C\left(\theta_{0}\right) N K^{-3 / 2}|\vec{k}|^{-1 / 2}\left(\left|\kappa\left(e^{-\theta}|\vec{k}| / K\right)\right|+\alpha|\vec{k}|\left|\kappa\left(e^{-\theta}|\vec{k}| / K\right)\right|\right),
$$

for some constant $C\left(\theta_{0}\right) \geq 0$. The rapid decay of $\kappa$ implies that

$$
\Lambda_{\beta}:=\left(\int J(\vec{k})^{2} \omega(\vec{k})^{\beta} d^{3} k\right)^{1 / 2}<\infty,
$$

for any $\beta>-2$. In particular, $\Lambda_{\beta}$ is uniform in $K \geq 1$, for any $1 \geq \beta>-2$. This uniformity in $K \geq 1$ is actually the basic requirement that determines $p=3 / 2$ in the coupling parameter $g=\alpha^{3 / 2} K^{p}$.

The main relative bound that we use is described in the following lemma.

Lemma I.1. For all $m, n \in \mathbb{N}_{0}$ with $1 \leq m+n \leq 2$ and all $\theta \in D\left(0, \theta_{0}\right)$, the operators $W_{m, n}(\theta)$ are defined on $\mathcal{D}\left(H_{0}\right)$ and obey the bound

$$
\left\|W_{m, n}(\theta)\left|H_{0}+i C\left(N, \theta_{0}\right)\right|^{-1}\right\| \leq\left(4\left(1+\Lambda_{0}^{2}+\Lambda_{-1}^{2}\right)\right)^{(m+n) / 2},
$$

for some constant $C(N, \theta)>0$.

Proof: We first note that the canonical commutation relations (I.8) allow us to convert estimates on $W_{1,0}(\theta)$ into those for $W_{0,1}(\bar{\theta})$. Indeed, for any $\psi \in \mathcal{D}\left(H_{0}\right)$,

$$
\begin{aligned}
\left\|W_{1,0}(\theta) \psi\right\|^{2} & =\left\|W_{0,1}(\bar{\theta}) \psi\right\|^{2}+\sum_{\lambda=1,2} \int d^{3} k\left\|w_{1,0}(\vec{k}, \lambda ; \theta) \psi\right\|^{2} \\
& \leq\left\|W_{0,1}(\bar{\theta}) \psi\right\|^{2}+2 \Lambda_{0}\left\|\left(-\Delta_{x}+1\right)^{1 / 2} \psi\right\|^{2}
\end{aligned}
$$


Furthermore,

$$
\begin{aligned}
\left\|W_{0,1}(\theta) \psi\right\| & \leq \sum_{\lambda=1,2} \int d^{3} k J(\vec{k})\left\|\left(-\Delta_{x}+1\right)^{1 / 2} a_{\lambda}(k) \psi\right\| \\
& \leq 2 \Lambda_{-1}\left\|\left(-\Delta_{x}+1\right)^{1 / 2} H_{f}^{1 / 2} \psi\right\|
\end{aligned}
$$

and hence

$$
\left\|W_{1,0}(\theta) \psi\right\|, \quad\left\|W_{0,1}(\theta) \psi\right\| \leq 2\left(\Lambda_{0}+\Lambda_{-1}\right)\left\|\left(-\Delta_{x}+H_{f}+1\right) \psi\right\| .
$$

Similarly, we convert estimates on $W_{2,0}$ and $W_{1,1}$ into those for $W_{0,2}$. For $W_{2,0}$, for instance, the canonical commutation relations (I.8) imply that

$$
\begin{aligned}
W_{2,0}(\theta)^{*} W_{2,0}(\theta)= & W_{0,2}(\bar{\theta})^{*} W_{0,2}(\bar{\theta}) \\
& +2 \int d \xi_{1} d \xi_{2} w_{2,0}\left(\xi_{1} ; \xi_{2} ; \theta\right)^{*} w_{2,0}\left(\xi_{1} ; \xi_{2} ; \theta\right) \\
& +4 \int d \xi_{1} d \xi_{2} d \xi_{3} w_{2,0}\left(\xi_{1} ; \xi_{3} ; \theta\right)^{*} w_{2,0}\left(\xi_{2} ; \xi_{3} ; \theta\right) a^{*}\left(\xi_{1}\right) a\left(\xi_{2}\right)
\end{aligned}
$$

where we denoted $\xi:=(\vec{k}, \lambda), \int d \xi:=\sum_{\lambda=1,2} \int d^{3} k$, and $a^{\#}(\xi):=a_{\lambda}^{\#}(\vec{k})$. This yields

$$
\left\|W_{2,0}(\theta) \psi\right\| \leq 3\left\|W_{0,2}(\bar{\theta}) \psi\right\|+4 \Lambda_{0}^{2}\|\psi\|^{2},
$$

and, as in (I.48), we obtain that

$$
\left\|W_{0,2}(\theta) \psi\right\| \leq \Lambda_{-1}^{2}\left\|H_{f} \psi\right\| \leq \Lambda_{-1}^{2}\left\|\left(-\Delta_{x}+H_{f}+1\right) \psi\right\| .
$$

The bound (I.46) follows from (I.49), (I.51), (I.52), and the additional use of the fact that $-\Delta_{x}$ is relatively $H_{e l}$-bounded with relative bound arbitrarily close to 1.

Now self-adjointness of $H_{g}$ on $\mathcal{D}\left(H_{0}\right)$ and dilatation analyticity of $W_{g}(\theta)$ are just two immediate implications of Lemma I.1.

Corollary I.2. If $0<g=(\alpha K)^{3 / 2}<\left(1+\Lambda_{0}^{2}+\Lambda_{-1}^{2}\right)^{-1 / 2} / 10$ then $H_{g}$ is self-adjoint and semibounded on $\mathcal{D}\left(H_{0}\right)$.

Corollary I.3. The map $W_{g}: D\left(0, \theta_{0}\right) \rightarrow \mathcal{B}\left(\mathcal{D}\left(H_{0}\right) ; \mathcal{H}\right), \theta \mapsto W_{g}(\theta)$ is analytic.

Finally, we establish the dilatation analyticity of $H_{g}(\theta):=U_{\theta} H_{g} U_{\theta}^{-1}$, assuming that the potential $V_{c}(x)$ is dilatation analytic, i.e., $D\left(0, \theta_{0}\right) \ni \theta \rightarrow$ $V_{c}\left(e^{\theta} x\right) \in \mathcal{B}\left(\mathcal{D}\left(H_{0}\right) ; \mathcal{H}\right)$ is an analytic function. This property holds in case 
that $H_{e l}=-\Delta_{x}+V_{c}(x)$ is the Hamiltonian of an atom, i.e., $M=1$, for arbitrary $\theta$. Indeed, in the atomic case we may choose without loss of generality the position of the nucleus to be the origin of the one-electron configuration space, and then we obtain

$$
H_{e l}(\theta):=U_{\theta} H_{e l} U_{\theta}^{-1}=e^{-2 \theta}\left(-\Delta_{x}\right)+e^{-\theta} V_{c}(x) .
$$

Therefore,

$$
H_{0}(\theta):=U_{\theta} H_{0} U_{\theta}^{-1}=H_{e l}(\theta)+e^{-\theta} H_{f}
$$

is an analytic family of type A and, by Corollary I.3, so is $H_{g}(\theta)=H_{0}(\theta)+$ $W_{g}(\theta)$. We summarize this discussion and a simple consequence of (I.54) in the following corollary.

Corollary I.4. The family $\left\{H_{g}(\theta) \mid \theta \in D\left(0, \theta_{0}\right)\right\}$ is dilatation analytic, i.e., the map $H_{g}: D\left(0, \theta_{0}\right) \rightarrow \mathcal{B}\left(\mathcal{D}\left(H_{0}\right) ; \mathcal{H}\right), \theta \mapsto H_{g}(\theta)$ is analytic. Moreover, there exists a constant $b \geq 0$ such that

$$
\left\|\Delta H_{e l}(\theta)\left(H_{e l} \pm i\right)^{-1}\right\| \leq b|\theta|,
$$

where $\Delta H_{e l}(\theta):=H_{e l}(\theta)-H_{e l}$, for all $\theta \in D\left(0, \theta_{0}\right)$.

\section{Soft Photon Bound and Existence of a Ground State}

In this section we derive a new soft photon bound; see Inequalities (II.5)-(II.6) and Theorem II.3 below. It is taylored for the minimal coupling model, and we use this bound to prove the existence of a ground state.

Theorem II.1. There exists a constant $C\left(N, \Sigma-E_{0}\right) \geq 0$ such that, for all $0<g=(\alpha K)^{3 / 2} \leq C\left(N, \Sigma-E_{0}\right)$, the Hamiltonian $H_{g}$ has a ground state, i.e., $E_{0}(\alpha):=\inf \sigma\left(H_{g}\right)$ is an eigenvalue.

Proof: We introduce the notation $a^{\#}(\vec{F}):=\left(a^{\#}\left(F_{1}\right), a^{\#}\left(F_{2}\right), a^{\#}\left(F_{3}\right)\right)^{T}$, $a^{*}(F):=\sum_{\lambda=1,2} \int d^{3} k F(\vec{k}, \lambda) a_{\lambda}^{*}(\vec{k})$, and $a(F):=\sum_{\lambda=1,2} \int d^{3} k F(\vec{k}, \lambda)^{*} a_{\lambda}(\vec{k})$. Here, $F$ is a function on $\mathbb{R}^{3} \times \mathbb{Z}_{2}$ with values in the operators on $\mathcal{H}_{\text {el }}$ such that $\sum_{\lambda=1,2} \int d^{3} k\left\|F(\vec{k}, \lambda)\left(-\Delta_{x}+1\right)^{-1 / 2}\right\|^{2}<\infty$. We further denote $\Phi(F):=$ 
$a^{*}(F)+a(F), \Phi(\vec{F}):=a^{*}(\vec{F})+a(\vec{F})$, and $\vec{p}_{j}:=-i \vec{\nabla}_{\vec{x}_{j}}$. In this notation, the interaction $W_{g}$ reads

$$
W_{g}=\sum_{j=1}^{N}\left\{-2 g \Phi\left(\vec{G}_{\vec{x}_{j}}\right) \cdot \vec{p}_{j}+g^{2} \Phi\left(\vec{G}_{\vec{x}_{j}}\right)^{2}+\vec{\sigma}_{j} \cdot g \Phi\left(\vec{B}_{\vec{x}_{j}}\right)\right\},
$$

where $\vec{G}$ and $\vec{B}$ are defined in Eqns. (I.32), (I.33), and (I.39).

Next, we introduce an infrared regularization by switching off the interaction for photons of small momenta. Specifically, we pick a "photon mass", $m>0$, that is, we replace $\vec{G}_{\vec{x}}(\vec{k}, \lambda), \vec{B}_{\vec{x}}(\vec{k}, \lambda)$, and $W_{g}$ in (I.32), (I.33), and (II.1) by

$$
\begin{gathered}
\vec{G}_{\vec{x}}^{(m)}(\vec{k}, \lambda):=\chi[\omega(k) \geq m] \vec{G}_{\vec{x}}(\vec{k}, \lambda) \\
\vec{B}_{\vec{x}}^{(m)}(\vec{k}, \lambda):=\chi[\omega(k) \geq m] \vec{B}_{\vec{x}}(\vec{k}, \lambda) \\
W_{g}^{(m)}:=\sum_{j=1}^{N}\left\{-2 g \Phi\left(\vec{G}_{\vec{x}_{j}}^{(m)}\right) \cdot \vec{p}_{j}+g^{2} \Phi\left(\vec{G}_{\vec{x}_{j}}^{(m)}\right)^{2}+g \vec{\sigma}_{j} \cdot \Phi\left(\vec{B}_{\vec{x}_{j}}^{(m)}\right)\right\}
\end{gathered}
$$

and we denote $H_{g}^{(m)}:=H_{0}+W_{g}^{(m)}$ and $E_{0}^{(m)}(g):=\inf \sigma\left(H_{g}^{(m)}\right)$. We remark that $H_{g}^{(m)} \rightarrow H_{g}^{(0)}=H_{g}$ in norm resolvent sense, as $m \rightarrow 0$. This easily follows from an estimate similar to Lemma I.1 (see [5]). In Theorem II.2 below we show that, for $g=(\alpha K)^{3 / 2}$ sufficiently small, $H_{g}^{(m)}$ has a ground state, $\phi_{m}$, i.e., there exists a normalized solution of $H_{g}^{(m)} \phi_{m}=E_{0}^{(m)}(g) \phi_{m}$, for all $m>0$. Since $\left\|\phi_{m}\right\|=1$, the family $\left\{\phi_{m}\right\}_{m>0}$ contains a weakly convergent subsequence, $\left\{\phi_{m(n)}\right\}_{n \in \mathbb{N}}$, where $\lim _{n \rightarrow \infty} m(n)=0$. We put $\phi_{0}:=$ $w-\lim _{n \rightarrow \infty} \phi_{m(n)}$. Then one easily shows [5], that $\phi_{0} \in \mathcal{D}\left(H_{g}\right)$ and that $H_{g} \phi_{0}=E_{0}(\alpha) \phi_{0}$. To conclude, it remains to show that $\phi_{0} \neq 0$.

To show that $\phi_{0} \neq 0$, we employ a soft photon bound, as in [5]. There is an important difference, though. The soft photon bound in [5] estimated the photon number expectation $\left\langle\phi_{m} \mid N_{f} \phi_{m}\right\rangle$, where $N_{f}:=\sum_{\lambda=1,2} \int a_{\lambda}^{*}(\vec{k}) a_{\lambda}(\vec{k}) d^{3} k$, in terms of $\sup _{\vec{x}}\left\|\omega^{-1} \vec{G}_{\vec{x}}\right\|_{2}$. It was derived from a virial type argument, using the commutator of $a_{\lambda}(\vec{k})$ and $H_{g}$. This bound does not directly apply to the present problem because $\left\|\omega^{-1} \vec{G}_{\vec{x}}\right\|_{2}=\infty$, for all $\vec{x}$. Modifying the argument slightly by using the commutator of $a_{\lambda}(\vec{k})-i F_{x}(\vec{k}, \lambda)$ and $H_{g}$, for a suitably chosen $F_{x}$, we avoid the appearance of $\sup _{\vec{x}}\left\|\omega^{-1} \vec{G}_{\vec{x}}\right\|_{2}$ on the right side of the estimate, which we trade for a factor of $\left\||x| \phi_{m}\right\|$.

More precisely, in Theorem II.3 below we show that

$$
\left\langle\phi_{m} \mid N_{f} \phi_{m}\right\rangle \leq C_{1}(N) g^{2}\left\|(1+|x|) \phi_{m}\right\|^{2},
$$


for some constant $C_{1}(N) \geq 0$. In [5] we showed that $\phi_{m}$ is exponentially localized in the electron variables. More generally, there exists an $\varepsilon>0$ such that

$$
\left\|e^{\varepsilon|x|} \chi_{\Delta}\left(H_{g}^{(m)}\right)\right\| \leq C_{2}<\infty,
$$

where $\chi_{\Delta}\left(H_{g}^{(m)}\right)$ is the spectral projection onto $\Delta:=\left(-\infty,\left(\Sigma+E_{0}\right) / 2\right)$, provided that $g^{2}\left(1+\Sigma-E_{0}\right)$ is sufficiently small (with $C_{2}$ and $\varepsilon$ independent of $g$ ). Since $\phi_{m} \in \operatorname{Ran}\left\{\chi_{\Delta}\left(H_{g}^{(m)}\right)\right\}$, this implies the boundedness of $\left\||x| \phi_{m}\right\|$. Thus there exists a constant, $C_{3} \equiv C_{3}\left(N, \Sigma-E_{0}\right)$, such that

$$
\left\langle\phi_{m} \mid N_{f} \phi_{m}\right\rangle \leq C_{3} g^{2}
$$

for all $m>0$. Next, we introduce the projection $P_{e l}$ onto all bound states of $H_{e l}$ below $\frac{1}{2}\left(\Sigma+E_{0}\right)<\Sigma$. Note that $P_{e l}$ has finite rank and that $\left(\Sigma-E_{0}\right) P_{e l}^{\perp} \leq$ $2\left(H_{0}-E_{0}\right)$. The latter implies that

$$
\begin{aligned}
\left\langle\phi_{m} \mid P_{e l}^{\perp} \phi_{m}\right\rangle & \leq 2\left(\Sigma-E_{0}\right)^{-1}\left\langle\phi_{m} \mid\left(E_{0}^{(m)}(g)-E_{0}-W_{g}^{(m)}\right) \phi_{m}\right\rangle \\
& \leq C_{3} g^{2}
\end{aligned}
$$

From (II.7)-(II.8) and $P_{e l} P_{\Omega} \geq 1-P_{e l}^{\perp}-N_{f}$ we draw the important consequence that

$$
\left\langle\phi_{m} \mid P_{e l} P_{\Omega} \phi_{m}\right\rangle \geq 1-2 C_{3} g^{2}
$$

where $P_{\Omega}:=|\Omega\rangle\langle\Omega|$ denotes the rank-one projection onto the photon vacuum vector $\Omega$. Thus, if $g=(\alpha K)^{3 / 2}$ is sufficiently small then $\left\langle\phi_{m} \mid P_{e l} P_{\Omega} \phi_{m}\right\rangle \geq$ $1 / 2$, for all $m>0$. Since $P_{e l} P_{\Omega}$ has finite rank, it follows that $\phi_{0} \neq 0$.

In the following theorem, we review the proof in [5] of the existence of a ground state of $H_{g}^{(m)}$, for $m>0$.

Theorem II.2. There exists a constant $C \equiv C\left(N, \Sigma-E_{0}\right)>0$ such that, for all $0<g=(\alpha K)^{3 / 2} \leq C$, the Hamiltonian $H_{g}^{(m)}$ has a ground state, i.e., $E_{0}^{(m)}(g):=\inf \sigma\left(H_{g}^{(m)}\right)$ is an eigenvalue, for any $m>0$.

Proof: We only sketch the argument, see [5, Section II.2] for details. Alternatively, one may proceed as in [16]. The assertion is proven if we can find some $\tilde{m}>0$ such that the sum of the negative eigenvalues of $H_{g}^{(m)}-$ $E_{0}^{(m)}(g)-\tilde{m}$ is finite, i.e., $\operatorname{Tr}\left\{\left[H_{g}^{(m)}-E_{0}^{(m)}(g)-\tilde{m}\right]_{-}\right\}>-\infty$, where the negative part of a real number $\lambda$ is defined as $[\lambda]_{-}:=\min \{\lambda, 0\}$. To this 
end, we first employ a discretization. Given $\varepsilon>0$ and a locally integrable function $F$, we define its $\varepsilon$-average by

$$
\langle F(\vec{k})\rangle_{\varepsilon}:=\varepsilon^{-3} \int_{\vec{n}(\vec{k})+Q_{\varepsilon}} F\left(\vec{k}^{\prime}, \lambda\right) d^{3} k^{\prime}
$$

where $Q_{\varepsilon}=[-\varepsilon / 2, \varepsilon / 2)^{3}$ and $\vec{n}(\vec{k}) \in(\varepsilon \mathbb{Z})^{3}$ is the integer part of $\vec{k}$, i.e., $\vec{k}-\vec{n}(\vec{k}) \in Q_{\varepsilon}$. We define the corresponding interaction, $W_{g}^{(m, \varepsilon)}$, by replacing $\vec{G}_{\vec{x}}^{(m)}(\vec{k}, \lambda)$ and $\vec{B}_{\vec{x}}^{(m)}(\vec{k}, \lambda)$ in (II.2)-(II.4) by $\left\langle\vec{G}_{\vec{x}}^{(m)}(\vec{k}, \lambda)\right\rangle_{\varepsilon}$ and $\left\langle\vec{B}_{\vec{x}}^{(m)}(\vec{k}, \lambda)\right\rangle_{\varepsilon}$, respectively. Then, by the bound (I.46) it follows that

$$
\begin{aligned}
& \pm\left(W_{g}^{(m, \varepsilon)}-W_{g}^{(m)}\right) \\
& \leq o\left(\varepsilon^{0}\right)\left(H_{g}^{(m)}-E_{0}^{(m)}(g)+1\right)^{1 / 2}(1+|x|)\left(H_{g}^{(m)}-E_{0}^{(m)}(g)+1\right)^{1 / 2},
\end{aligned}
$$

where $o\left(\varepsilon^{0}\right)$ denotes a function which possibly depends on $g, N, \kappa, m$ and tends to zero as $\varepsilon \rightarrow 0$. Here, our original manuscript contained a small mistake in that the factor $1+|x|$ was missing, as was kindly pointed out to us by F. Hiroshima. Next, we define $H_{f}^{(m, \varepsilon)}$ by replacing $\omega(\vec{k})$ in $H_{f}^{(m)}$ by $\langle\omega(\vec{k})\rangle_{\varepsilon}$. Since $\left|\omega(\vec{k})-\langle\omega(\vec{k})\rangle_{\varepsilon}\right|=O(\varepsilon)$ and $\omega(k) \geq m$, we obtain that

$$
\left(1-o\left(\varepsilon^{0}\right)\right) H_{f}^{(m)} \leq H_{f}^{(m, \varepsilon)} \leq\left(1+o\left(\varepsilon^{0}\right)\right) H_{f}^{(m)} .
$$

Denoting $H_{g}^{(m, \varepsilon)}:=H_{e l}+H_{f}^{(m, \varepsilon)}+W_{g}^{(m, \varepsilon)}$, we hence obtain that

$$
\begin{aligned}
H_{g}^{(m)} \geq & \left(1-o\left(\varepsilon^{0}\right)\right) H_{g}^{(m, \varepsilon)} \\
& -o\left(\varepsilon^{0}\right)\left(H_{g}^{(m)}-E_{0}^{(m)}(g)+1\right)^{1 / 2}(1+|x|)\left(H_{g}^{(m)}-E_{0}^{(m)}(g)+1\right)^{1 / 2} .
\end{aligned}
$$

Next, we introduce the interval $\widetilde{\Delta}:=\left(-\infty, E_{0}^{(m)}(g)+\tilde{m}\right)$ and observe that

$$
\left[H_{g}^{(m)}-E_{0}^{(m)}(g)-\tilde{m}\right]_{-}=\chi_{\widetilde{\Delta}}\left(H_{g}^{(m)}\right)\left(H_{g}^{(m)}-E_{0}^{(m)}(g)-\tilde{m}\right) \chi_{\widetilde{\Delta}}\left(H_{g}^{(m)}\right) .
$$

Furthermore, we note that, thanks to (II.6) and $\chi_{\widetilde{\Delta}}=\chi_{\widetilde{\Delta}} \chi_{\Delta}$, for $\tilde{m}>0$ sufficiently small, we have

$$
\begin{aligned}
& \| \chi_{\widetilde{\Delta}}\left(H_{g}^{(m)}\right)\left(H_{g}^{(m)}-E_{0}^{(m)}(g)+1\right)^{1 / 2} \\
& \quad(1+|x|)\left(H_{g}^{(m)}-E_{0}^{(m)}(g)+1\right)^{1 / 2} \chi_{\widetilde{\Delta}}\left(H_{g}^{(m)}\right) \|<\infty .
\end{aligned}
$$


Thus,

$$
\begin{aligned}
& \operatorname{Tr}\left\{\left[H_{g}^{(m)}-E_{0}^{(m)}(g)-\tilde{m}\right]_{-}\right\} \\
& \geq \operatorname{Tr}\left\{\chi_{\widetilde{\Delta}}\left(H_{g}^{(m)}\right)\left(H_{g}^{(m, \varepsilon)}-E_{0}^{(m, \varepsilon)}(g)-\tilde{m}-o\left(\varepsilon^{0}\right)\right) \chi_{\widetilde{\Delta}}\left(H_{g}^{(m)}\right)\right\} \\
& \geq \operatorname{Tr}\left\{\chi_{\widetilde{\Delta}}\left(H_{g}^{(m)}\right)\left[H_{g}^{(m, \varepsilon)}-E_{0}^{(m, \varepsilon)}(g)-\tilde{m}-o\left(\varepsilon^{0}\right)\right]_{-} \chi_{\widetilde{\Delta}}\left(H_{g}^{(m)}\right)\right\} \\
& \geq \operatorname{Tr}\left\{\left[H_{g}^{(m, \varepsilon)}-E_{0}^{(m, \varepsilon)}(g)-\tilde{m} / 2\right]_{-}\right\},
\end{aligned}
$$

for $\varepsilon>0$ sufficiently small, and the finiteness of the right side in (II.16) (and hence the claim) follows if we can show that, for any $\varepsilon>0$, the discretized Hamiltonian $H_{g}^{(m, \varepsilon)}$ has only finitely many eigenvalues below $E_{0}^{(m, \varepsilon)}(g)+\tilde{m} / 2$.

The key point of the discretization by means of the $\varepsilon$-average is the tensor product representation $\mathcal{F} \cong \mathcal{F}\left[\mathcal{H}_{\text {disc }}\right] \otimes \mathcal{F}\left[\mathcal{H}_{\text {disc }}^{\perp}\right]$, where $\mathcal{H}_{\text {disc }}$ is spanned by $\chi_{Q_{\varepsilon}+\vec{n}}, \vec{n} \in(\varepsilon \mathbb{Z})^{3}$ (see [5] for details). Note that, with respect to this representation, we have $H_{f}^{(m, \varepsilon)} \cong H_{f}^{(m, \varepsilon)} \otimes \mathbf{1}+\mathbf{1} \otimes H_{f}^{(m, \varepsilon)}, W_{g}^{(m, \varepsilon)} \cong W_{g}^{(m, \varepsilon)} \otimes \mathbf{1}$, and hence

$$
\begin{aligned}
H_{g}^{(m, \varepsilon)} & \cong H_{g}^{(m, \varepsilon)} \otimes \mathbf{1}+\mathbf{1} \otimes H_{f}^{(m, \varepsilon)} \\
& \geq\left(E_{0}^{(m, \varepsilon)}(g)+m\right) \otimes P_{\Omega}^{\perp}+H_{g}^{(m, \varepsilon)} \otimes P_{\Omega},
\end{aligned}
$$

denoting the projection onto the vacuum in $\mathcal{F}\left[\mathcal{H}_{\text {disc }}^{\perp}\right]$ by $P_{\Omega}$. Hence, for $\tilde{m} \leq m$,

$$
H_{g}^{(m, \varepsilon)}-E_{0}^{(m, \varepsilon)}(g)-\tilde{m} / 2 \geq\left(H_{g}^{(m, \varepsilon)}-E_{0}^{(m, \varepsilon)}(g)-\tilde{m} / 2\right) \otimes P_{\Omega} .
$$

Next, for sufficiently small $\varepsilon>0$, an estimate similar to Lemma I.1 together with an interpolation argument implies that, on $\mathcal{H}_{e l} \otimes \mathcal{F}\left[\mathcal{H}_{\text {disc }}\right]$, we have

$$
W_{g}^{(m, \varepsilon)} \geq-C_{1}^{\prime} g\left(H_{e l}+H_{f}^{(m, \varepsilon)}+C(N)\right),
$$

for some constants $C_{1}^{\prime}, C(N) \geq 0$. Thus, we obtain that

$$
\begin{aligned}
& H_{g}^{(m, \varepsilon)}-E_{0}^{(m, \varepsilon)}(g) \\
& \geq \quad\left(1-C_{1}^{\prime} g\right) H_{e l}-\left(1+C_{1}^{\prime} g\right) E_{0}+\left(1-C_{1}^{\prime} g\right) H_{f}^{(m, \varepsilon)}-2 C_{1}^{\prime} C(N) \\
& \geq\left\{\frac{1}{2}\left(\Sigma-E_{0}\right)-2 C_{1}^{\prime} g\left(1+2\left|E_{0}\right|\right)-o\left(\varepsilon^{0}\right)\right\} P_{e l}^{\perp} \\
& \quad+\left\{\left(1-C_{1}^{\prime} g\right) H_{f}^{(m, \varepsilon)}-2 C_{1}^{\prime} g\left(1+2\left|E_{0}\right|\right)-o\left(\varepsilon^{0}\right)\right\} P_{e l},
\end{aligned}
$$

where $P_{e l}$ is the finite dimensional projection onto the bound states of $H_{e l}$ of energy $<\frac{1}{2}\left(\Sigma+E_{0}\right)$. Now, if $g$ is sufficiently small such that

$$
\hat{m}:=\frac{1}{2}\left(\Sigma-E_{0}\right)-2 C_{1}^{\prime} g\left(1+2\left|E_{0}\right|\right)>0
$$


then, for any $0<\tilde{m}<\min \{m, \hat{m}\}$, we obtain from inserting (II.20) into (II.18) that

$$
\begin{aligned}
& H_{g}^{(m)}-E_{0}^{(m)}(g)-\tilde{m} / 2 \\
& \geq\left\{\left(1-o\left(\varepsilon^{0}\right)-2 C_{1}^{\prime} g\right) H_{f}^{(m, \varepsilon)}-2 C_{1}^{\prime} g\left(1+2\left|E_{0}\right|\right)-o\left(\varepsilon^{0}\right)\right\} P_{e l} \otimes P_{\Omega},
\end{aligned}
$$

for $\varepsilon>0$ sufficiently small. The right side, however, has clearly only finitely many negative eigenvalues, for any $\varepsilon>0$, which proves that, for any $\varepsilon>0$, the discretized Hamiltonian $H_{g}^{(m, \varepsilon)}$ has only finitely many eigenvalues below $E_{0}^{(m, \varepsilon)}(g)+\tilde{m} / 2$.

Theorem II.3. Let $\phi_{m}$ be a ground state of $H_{g}^{(m)}$, for $m>0$, and denote by $N_{f}:=\sum_{\lambda=1,2} \int a_{\lambda}^{*}(\vec{k}) a_{\lambda}(\vec{k}) d^{3} k$ the photon number operator. Assume that $\alpha(K+1) \leq 1$. Then there exist constants $C(N), C_{1}(N) \geq 0$ such that

$$
\begin{gathered}
\left\langle\phi_{m} \mid N_{f} \phi_{m}\right\rangle \leq C(N) g^{2} \sup _{\vec{x}, \lambda}\left\{\int \operatorname{Tr}_{3 \times 3}\left(\left|\vec{\nabla}_{\vec{x}} \vec{G}_{\vec{x}}(\vec{k}, \lambda)\right|^{2}\right)\left(1+\frac{1}{\omega(\vec{k})^{2}}\right) d^{3} k\right. \\
\left.+\int\left|\vec{G}_{\vec{x}}(\vec{k}, \lambda)\right|^{2} d^{3} k+\int\left(\left|\vec{B}_{\vec{x}}(\vec{k}, \lambda)\right|^{2}+\left|\Delta_{\vec{x}} \vec{G}_{\vec{x}}(\vec{k}, \lambda)\right|^{2}\right) \frac{d^{3} k}{\omega(\vec{k})^{2}}\right\}\left\|(1+|x|) \phi_{m}\right\|^{2} \\
\leq C_{1}(N) g^{2}\left\|(1+|x|) \phi_{m}\right\|^{2}
\end{gathered}
$$

Before giving the proof of Theorem II.3, we remark that using the definitions (I.32), (I.33), and (I.39) of $\vec{G}$ and $\vec{B}$, one easily checks the integrals on the right side of Eqn. (II.23) to be bounded by a constant, uniformly in $K \geq 1$.

Proof of Theorem II.3: Throughout the proof we omit the superscript " $(m)$ ". To prove the asserted bound, we first observe the following commutation relations,

$$
\begin{aligned}
a_{\lambda}(\vec{k}) H_{g}= & \left(H_{g}+\omega(\vec{k})\right) a_{\lambda}(\vec{k}) \\
& +\sum_{j=1}^{N}\left\{\vec{\sigma}_{j} \cdot g \vec{B}_{\vec{x}_{j}}(\vec{k}, \lambda)-2 g \vec{G}_{\vec{x}_{j}}(\vec{k}, \lambda)\right\} \cdot\left(\vec{p}_{j}-g \Phi\left(\vec{G}_{\vec{x}_{j}}\right)\right), \\
F_{\vec{x}_{j}}(\vec{k}, \lambda) H_{g}= & H_{g} F_{\vec{x}_{j}}(\vec{k}, \lambda) \\
& +\Delta_{\vec{x}_{j}} F_{\vec{x}_{j}}(\vec{k}, \lambda)+2 i \vec{\nabla}_{\vec{x}_{j}} F_{\vec{x}_{j}}(\vec{k}, \lambda) \cdot\left(\vec{p}_{j}-g \Phi\left(\vec{G}_{\vec{x}_{j}}\right)\right),
\end{aligned}
$$


on $C_{0}\left(\mathbb{R}^{3 N} ; \mathcal{F}\right) \cap \mathcal{D}\left(H_{0}\right)$, for any $F \in C^{2}\left(\mathbb{R}^{3} ; L^{2}\left(\mathbb{R}^{3} \times \mathbb{Z}_{2}\right)\right)$. We therefore have

$$
\begin{aligned}
& \left(a_{\lambda}(\vec{k})-i \sum_{j=1}^{N} F_{\vec{x}_{j}}(\vec{k}, \lambda)\right)\left(H_{g}-E_{0}(\alpha)\right) \\
& =\left(H_{g}-E_{0}(\alpha)+\omega(\vec{k})\right) a_{\lambda}(\vec{k})-i\left(H_{g}-E_{0}(\alpha)\right)\left(\sum_{j=1}^{N} F_{\vec{x}_{j}}(\vec{k}, \lambda)\right) \\
& \quad+\sum_{j=1}^{N}\left\{\vec{\sigma}_{j} \cdot g \vec{B}_{\vec{x}_{j}}(\vec{k}, \lambda)+\Delta_{\vec{x}_{j}} F_{\vec{x}_{j}}(\vec{k}, \lambda)\right. \\
& \left.\quad-2\left(\vec{p}_{j}-g \Phi\left(\vec{G}_{\vec{x}_{j}}\right)\right) \cdot\left(g \vec{G}_{\vec{x}_{j}}(\vec{k}, \lambda)-\vec{\nabla}_{\vec{x}_{j}} F_{\vec{x}_{j}}(\vec{k}, \lambda)\right)\right\}
\end{aligned}
$$

We apply (II.26) to $\chi(|x| / R) \phi_{m}$, where $\chi \in C_{0}^{\infty}(\mathbb{R} ;[0,1])$ and $\equiv 1$ on $[-1,1]$. Letting $R \rightarrow \infty$ and using $\left(H_{g}-E_{0}(\alpha)\right) \phi_{m}=0$, we derive that

$$
\begin{aligned}
a_{\lambda}(\vec{k}) \phi_{m}= & i R(\omega(k))\left(H_{g}-E_{0}(\alpha)\right)\left(\sum_{j=1}^{N} F_{\vec{x}_{j}}(\vec{k}, \lambda)\right) \phi_{m} \\
+ & R(\omega(k)) \sum_{j=1}^{N}\left\{\vec{\sigma}_{j} \cdot g \vec{B}_{\vec{x}_{j}}(\vec{k}, \lambda)+\Delta_{\vec{x}_{j}} F_{\vec{x}_{j}}(\vec{k}, \lambda)\right. \\
& \left.-2\left(\vec{p}_{j}-g \Phi\left(\vec{G}_{\vec{x}_{j}}\right)\right) \cdot\left(g \vec{G}_{\vec{x}_{j}}(\vec{k}, \lambda)-\vec{\nabla}_{\vec{x}_{j}} F_{\vec{x}_{j}}(\vec{k}, \lambda)\right)\right\} \phi_{m}
\end{aligned}
$$

where $R(\omega):=\left(H_{g}-E_{0}(\alpha)+\omega\right)^{-1}$. We choose $F_{\vec{x}}(\vec{k}, \lambda):=g \vec{x} \cdot \vec{G}_{\vec{x}}(\vec{k}, \lambda)$. Then (II.27) reads

$$
\begin{aligned}
& a_{\lambda}(\vec{k}) \phi_{m}= i g R(\omega(k)) \\
&+R(\omega(k)) \sum_{j=1}^{N}\left\{\vec{\sigma}_{j} \cdot g \vec{B}_{\vec{x}_{j}}(\vec{k}, \lambda)+i g \vec{x}_{j} \cdot \Delta_{\vec{x}_{j}} \vec{G}_{\vec{x}_{j}}(\vec{k}, \lambda)\left(\sum_{j=1}^{N} \vec{x}_{j} \cdot \vec{G}_{\vec{x}_{j}}(\vec{k}, \lambda)\right) \phi_{m}\right. \\
&\left.+2 g\left[\left(\left(\vec{p}_{j}-g \Phi\left(\vec{G}_{\vec{x}_{j}}\right)\right) \cdot \vec{\nabla}_{\vec{x}_{j}}\right) \vec{G}_{\vec{x}_{j}}(\vec{k}, \lambda)\right] \cdot \vec{x}_{j}\right\} \phi_{m} .
\end{aligned}
$$

We observe that $\left\|R(\omega(k))\left(H_{g}-E_{0}(\alpha)\right)\right\| \leq 1$ and that $\left|\sum_{j=1}^{N} \vec{x}_{j} \cdot \vec{G}_{\vec{x}_{j}}\right| \leq$ $|x|\left(\sum_{j=1}^{N}\left|\vec{G}_{\vec{x}_{j}}\right|^{2}\right)^{1 / 2}$, denoting $|x|^{2}:=\sum_{j=1}^{N}\left(\vec{x}_{j}\right)^{2}$. Hence, for $\lambda \in\{1,2\}$,

$$
\begin{aligned}
\int d^{3} k \| R(\omega(k))\left(H_{g}\right. & \left.-E_{0}(\alpha)\right) \sum_{j=1}^{N} \vec{x}_{j} \cdot \vec{G}_{\vec{x}_{j}}(\vec{k}, \lambda) \phi_{m} \| \\
& \leq N \sup _{\vec{x}}\left\{\int\left|\vec{G}_{\vec{x}}(\vec{k}, \lambda)\right|^{2} d^{3} k\right\}^{1 / 2}\left\||x| \phi_{m}\right\| .
\end{aligned}
$$


Additionally using $\|R(\omega(k))\| \leq \omega(\vec{k})^{-1}$, we similarly obtain

$$
\begin{aligned}
& \int d^{3} k\left\|R(\omega(k)) \sum_{j=1}^{N}\left\{\vec{\sigma}_{j} \cdot \vec{B}_{\vec{x}_{j}}(\vec{k}, \lambda)+i \vec{x}_{j} \cdot \Delta_{\vec{x}_{j}} \vec{G}_{\vec{x}_{j}}(\vec{k}, \lambda)\right\} \phi_{m}\right\| \\
& \leq N \sup _{\vec{x}}\left\{\int\left(\left|\vec{B}_{\vec{x}}(\vec{k}, \lambda)\right|^{2}+\left|\Delta_{\vec{x}} \vec{G}_{\vec{x}}(\vec{k}, \lambda)\right|^{2}\right) \frac{d^{3} k}{\omega(\vec{k})^{2}}\right\}^{1 / 2}\left\|(1+|x|) \phi_{m}\right\| .
\end{aligned}
$$

Furthermore, we note that

$$
\begin{aligned}
\left\|R(\omega(k))\left(H_{g}-E_{0}(\alpha)+1\right)\right\| & \leq\left(1+\omega(\vec{k})^{-1}\right), \\
\left\|R(\omega(k)) \sum_{j=1}^{N}\left(\vec{p}_{j}-g \Phi\left(\vec{G}_{\vec{x}_{j}}\right)\right)^{2}\right\| & \leq C(N),
\end{aligned}
$$

for some constant $C(N) \geq 0$ which only depends on $N$. These bounds yield the following estimate,

$$
\begin{aligned}
\int d^{3} k \| & \left.R(\omega(k)) \sum_{j=1}^{N}\left[\left(\left(\vec{p}_{j}-g \Phi\left(\vec{G}_{\vec{x}_{j}}\right)\right) \cdot \vec{\nabla}_{\vec{x}_{j}}\right) \vec{G}_{\vec{x}_{j}}(\vec{k}, \lambda)\right] \cdot \vec{x}_{j}\right\} \phi_{m} \| \\
\leq & N \sup _{\vec{x}}\left\{\int \operatorname{Tr}_{3 \times 3}\left(\left|\vec{\nabla}_{\vec{x}} \vec{G}_{\vec{x}}(\vec{k}, \lambda)\right|^{2}\right)\left(1+\omega(\vec{k})^{-2}\right) d^{3} k\right\}^{1 / 2}\left\||x| \phi_{m}\right\| .
\end{aligned}
$$

Thus we arrive at the assertion.

\section{Resonances and Time-Decay Estimates}

In the present section, we study spectral properties of the Hamiltonian $H_{g}$. We also study the propagator $\exp \left[-i t H_{g}\right]$ applied to states whose spectral support is localized about the excited atomic energy level $E_{j}, j \geq 1$. As we describe in the introduction, our main tool for this analysis is the complex dilatation, $H_{g}(\theta)=U_{\theta} H_{g} U_{\theta}^{-1}$, of the Hamiltonian $H_{g}$, where $U_{\theta}$ is the dilatation defined in (I.18). We prove below that, for $\theta=i \vartheta, 0<\vartheta$, and $g \ll \vartheta$, a complex neighbourhood of an interval $\mathcal{I}_{j}$ about $E_{j}$ does not contain any spectrum of $H_{g}(\theta)$. By the dilatation analyticity of $H_{g}(\theta)$ in $\theta$, this implies that the spectrum of $H_{g}$ in $\mathcal{I}_{j}$ is purely absolutely continuous, and it allows for an estimate of the time decay rate of certain states in $\operatorname{Ran}\left\{\chi_{\mathcal{I}_{j}}\left(H_{g}\right)\right\}$. 
To state this estimate more precisely, we recall from (I.32)-(I.38) that

$$
\begin{aligned}
H_{g}(\theta) & =H_{0}(\theta)+W_{g}(\theta) \\
H_{0}(\theta) & =H_{e l}(\theta)+e^{-\theta} H_{f}, \\
W_{g}(\theta) & =\sum_{m+n=1}^{2} g^{m+n} W_{m, n}(\theta)+g^{2} C_{n o},
\end{aligned}
$$

where $g:=(\alpha K)^{3 / 2}$, and $C_{n o}:=2 N \int\left|\vec{G}_{\vec{x}}(\vec{k}, 1)\right|^{2} d^{3} k$ is an energy shift resulting from normal-ordering $W_{g}$. We absorb this constant by redefining

$$
W_{g}(\theta) \rightarrow W_{g}(\theta)+g^{2} C_{n o} \quad, \quad H_{e l}(\theta) \rightarrow H_{e l}(\theta)-g^{2} C_{n o},
$$

and since it only shifts all energies by $C_{n o}$, we henceforth ignore the constant $C_{n o}$ by setting it equal to zero. Thus we obtain

$$
H_{g}(\theta):=U_{\theta} H_{g} U_{\theta}^{-1}=H_{0}(\theta)+W_{g}(\theta)=H_{0}(\theta)+\sum_{m+n \leq 2} g^{m+n} W_{m, n}(\theta) .
$$

Recall that we assumed in Eqn. (I.14) that the $j^{\text {th }}$ atomic energy level is of finite degeneracy $n_{j}$ and isolated from the rest of the spectrum of $H_{e l}$ by a positive distance

$$
\delta:=\operatorname{dist}\left\{E_{j}, \sigma\left(H_{e l}\right) \backslash\left\{E_{j}\right\}\right\}>0 .
$$

As in [5, Eqns. (IV.84), (IV.85)], we define two $n_{j} \times n_{j}$ matrices by

$$
\begin{aligned}
Z_{j}^{\mathrm{od}}(\theta):= & \sum_{\lambda=1,2} \int U_{\theta} P_{e l, j} w_{0,1}(\vec{k}, \lambda) P_{e l, j}^{\perp} \\
& \left(H_{e l}-E_{j}+\omega(\vec{k})-i 0\right)^{-1} P_{e l, j}^{\perp} w_{1,0}(\vec{k}, \lambda) P_{e l, j} U_{\theta}^{-1} d^{3} k, \\
Z_{j}^{\mathrm{d}}(\theta):= & \sum_{\lambda=1,2} \int U_{\theta} P_{e l, j} w_{0,1}(\vec{k}, \lambda) P_{e l, j} w_{1,0}(\vec{k}, \lambda) P_{e l, j} U_{\theta}^{-1} \frac{d^{3} k}{\omega(\vec{k})},
\end{aligned}
$$

where $P_{e l, j}:=\sum_{\ell=1}^{n_{j}}\left|\varphi_{j, \ell}\right\rangle\left\langle\varphi_{j, \ell}\right|$ is the projection onto the eigenspace of $H_{e l}$ corresponding to the eigenvalue $E_{j}$. Note that the matrices $Z_{j}^{\text {od }}(\theta)$ and $Z_{j}^{\mathrm{d}}(\theta)$ are similar to $Z_{j}^{\text {od }}(0)$ and $Z_{j}^{\mathrm{d}}(0)$, respectively, for all $\theta \in D\left(0, \theta_{0}\right)$. We remark that $Z_{j}^{\mathrm{d}}(0)$ and $\operatorname{Im}\left\{Z_{j}^{\text {od }}(0)\right\} \equiv\left(Z_{j}^{\text {od }}(0)-Z_{j}^{\text {od }}(0)^{*}\right) / 2 i$ are self-adjoint. We require $\operatorname{Im}\left\{Z_{j}^{\text {od }}(\theta)\right\}$ to be invertible and to obey

$$
0<c \leq \Gamma_{j}:=\min \left\{\sigma\left(\operatorname{Im}\left\{Z_{j}^{\text {od }}(0)\right\}\right)\right\} \leq c^{\prime},
$$


for some constants $0<c \leq c^{\prime}$. Given a small constant $\varepsilon>0$, and a large constant $C>0$, we define the following union of "comet-shaped" sets $\mathcal{R}_{j} \equiv$ $\mathcal{R}_{j}(\varepsilon, C) \subseteq \mathbb{C}_{-}$in the lower halfplane,

$$
\mathcal{R}_{j}:=E_{j}+g^{2} \sigma\left(Z_{j}^{\mathrm{d}}(\theta)\right)-i g^{2} \Gamma_{j}+e^{-\theta} \mathbb{R}_{+}+D\left(0, C g^{2+\varepsilon}\right),
$$

Furthermore, for any $0<\rho<\delta$, we define the interval

$$
\mathcal{I}_{j}(\rho):=\left(E_{j}-\rho, E_{j}+\rho\right),
$$

and we remark that $\operatorname{dist}\left\{\mathcal{I}_{j}(\delta / 2), \sigma\left(H_{e l}\right) \backslash\left\{E_{j}\right\}\right\} \geq \delta / 2$.

Now we are ready to formulate the first main spectral result of this section

Theorem III.1. Let $0<\varepsilon<1 / 3$. For $\theta=i \vartheta$ and $\vartheta>0$ and $g>0$ sufficiently small, there exist constants $c, C>0$ such that

$$
\mathcal{A}_{j}:=\left(\mathcal{I}_{j}(\delta / 2)+i\left[-c g^{2-\varepsilon}, \infty\right)\right) \backslash \mathcal{R}_{j}(\varepsilon, C) \subseteq \rho\left(H_{g}(\theta)\right),
$$

where $\rho\left(H_{g}(\theta)\right)$ is the resolvent set of $H_{g}(\theta)$, and for any $z \in \mathcal{A}_{j}$, we have that

$$
\left\|\left(H_{g}(\theta)-z\right)^{-1}\right\| \leq C^{\prime} \operatorname{dist}\left\{z, \mathcal{R}_{j}\right\}^{-1},
$$

for some $C^{\prime}>0$.

Theorem III.1 has two important consequences. The first is immediate from the analytic continuation in $\theta$, and we state it as a corollary.

Corollary III.2. For $g>0$ sufficiently small, the spectrum of $H_{g}$ in $\mathcal{I}_{j}(\delta / 2)$ is absolutely continuous, $\mathcal{I}_{j}(\delta / 2) \subseteq \sigma_{\text {ac }}\left(H_{g}\right)$.

\section{III.1 Time-Decay Estimates}

To formulate the second main result of this section, another consequence of Theorem III.1, we pick a smooth function $F \in C_{0}^{\infty}([0,1 / 2) ;[0,1])$ such that $F \equiv 1$ on $[0,1 / 4]$, and we define $F_{j} \in C_{0}^{\infty}\left(\mathcal{I}_{j}(\delta / 2) ;[0,1]\right)$, with $F_{j} \equiv 1$ on $\mathcal{I}_{j}(\delta / 4)$, by $F_{j}(\lambda):=F\left(\delta^{-1}\left|\lambda-E_{j}\right|\right)$. Furthermore, for $\eta \in \mathcal{H}$, we set

$$
\psi_{j} \equiv \psi_{j}(\eta):=P_{e l, j} \exp \left[-g^{-2} H_{f}\right] \eta \text {. }
$$

Theorem III.3. Let $\eta \in \mathcal{H}$ be normalized, dilatation analytic in $D\left(0, \theta_{0}\right)$. Denote $\eta(\theta):=U_{\theta} \eta$, and assume that $B_{\eta}:=1+\sup _{|\theta| \leq \theta_{0}}\|\eta(\theta)\|<\infty$. Moreover, assume that $g>0$ is sufficiently small, $t>1$, and $0<\varepsilon<1 / 3$. 
Then there exist a constant $C \geq 0$ and, for any $N \in \mathbb{N}$, a constant $C_{N} \geq 0$ such that

$$
\begin{aligned}
& \left|\left\langle\psi_{j} \mid \exp \left[-i t H_{g}\right] F_{j}\left(H_{g}\right) \psi_{j}\right\rangle\right| \\
& \quad \leq B_{\eta}^{2}\left(C \ln (1 / g) \exp \left[-t\left(g^{2} \Gamma_{j}-C g^{2+\varepsilon}\right)\right]+C_{N} t^{-N} g^{4}\right) .
\end{aligned}
$$

Proof of Theorem III.3, given Theorem III.1: We first use the fact that

$$
F_{j}(\lambda)=-\delta^{-1} \int_{\delta / 4}^{\delta / 2} F^{\prime}(\omega / \delta) \chi_{\mathcal{I}_{j}(\omega)}(\lambda) d \omega
$$

to rewrite the matrix element on the left side of (III.15) as

$$
\begin{aligned}
& \left\langle\psi_{j} \mid \exp \left[-i t H_{g}\right] F_{j}\left(H_{g}\right) \psi_{j}\right\rangle \\
& =-\delta^{-1} \int_{\delta / 4}^{\delta / 2} F^{\prime}(\omega / \delta)\left\langle\psi_{j} \mid \exp \left[-i t H_{g}\right] \chi_{\mathcal{I}_{j}(\omega)}\left(H_{g}\right) \psi_{j}\right\rangle d \omega \\
& =\int_{\delta / 4}^{\delta / 2} \frac{F^{\prime}(\omega / \delta)}{\pi \delta} \int_{\mathcal{I}_{j}(\omega)} e^{-i t \lambda} \operatorname{Im}\left\{\left\langle\psi_{j}(\bar{\theta}) \mid\left(H_{g}(\theta)-\lambda\right)^{-1} \psi_{j}(\theta)\right\rangle\right\} d \lambda d \omega
\end{aligned}
$$

where we use Stone's formula (see, e.g., [26]) together with Theorem III.1, which implies that the $\operatorname{limit}_{\lim _{\varepsilon} \backslash 0}\left(H_{g}(\theta)-\lambda-i \varepsilon\right)^{-1}=\left(H_{g}(\theta)-\lambda\right)^{-1}$ exists and is bounded. Indeed, Theorem III.1 even implies that $\lambda-i s \mapsto\left(H_{g}(\theta)-\right.$ $\lambda+i s)^{-1}$ is bounded analytic, provided $s \in \mathbb{R}$ is not too large. We exploit this fact by deforming the integration contour $\mathcal{I}_{j}(\omega) \subseteq \mathbb{C}$ into the lower half-plane. To this end, we define a number

$$
S:=g^{2} \Gamma_{j}-C_{S} g^{2+\varepsilon},
$$

where $C_{S}>0$ is later chosen sufficiently large, and we assume that $\lambda \in$ $\mathcal{I}_{j}(\delta / 2)$ and $s \in\left[-S, g^{2}\right]$. Then, Theorem III.1 yields that

$$
\left\|\left(H_{g}(\theta)-\lambda-i s\right)^{-1}\right\| \leq C^{\prime}\left(\operatorname{dist}\left\{\lambda+i s, \mathcal{R}_{j}\right\}\right)^{-1},
$$

for $g=(\alpha K)^{3 / 2}$ sufficiently small and some $C^{\prime} \geq 0$, where $\mathcal{R}_{j}$ is defined in (III.10) above. Since $Z_{j}^{\mathrm{d}}(\theta)$ is similar to a self-adjoint $n_{j} \times n_{j}$-matrix, we may write

$$
\mathcal{R}_{j}:=\bigcup_{e \in \sigma\left(Z_{j}^{\mathrm{d}}(\theta)\right)} \mathcal{R}_{j}(e)
$$


with

$$
\mathcal{R}_{j}(e):=E_{j}+g^{2} e-i g^{2} \Gamma_{j}+e^{-\theta} \mathbb{R}_{+}+D\left(0, C g^{2+\varepsilon}\right) .
$$

Hence

$$
\begin{aligned}
& \left\|\left(H_{g}(\theta)-\lambda-i s\right)^{-1}\right\| \leq 2\left(\min _{e \in \sigma\left(Z_{j}^{\mathrm{d}}(\theta)\right)} \operatorname{dist}\left\{\lambda+i s, \mathcal{R}_{j}(e)\right\}\right)^{-1}(\text { III. } 22) \\
& \leq 2 \max _{e \in \sigma\left(Z_{j}^{\mathrm{d}}(\theta)\right)}\left[\operatorname{dist}\left\{\lambda-E_{j}-e g^{2}+i C_{S} g^{2+\varepsilon}, e^{-i \vartheta} \mathbb{R}_{+}\right\}-C g^{2+\varepsilon}\right]^{-1}
\end{aligned}
$$

where $C g^{2+\varepsilon}$ takes the disc $D\left(0, C g^{2+\varepsilon}\right)$ in the definition of $\mathcal{R}_{j}(e)$ into account. Thus, choosing $C_{S}$ in (III.18) larger than $4 C$, we obtain that

$$
\left\|\left(H_{g}(\theta)-\lambda-i s\right)^{-1}\right\| \leq \frac{2 \pi}{\vartheta} \max _{e \in \sigma\left(Z_{j}^{\mathrm{d}}(\theta)\right)}\left[\left(\lambda-E_{j}-e g^{2}\right)^{2}+\left(C_{S} g^{2+\varepsilon} / 4\right)^{2}\right]^{-1 / 2} .
$$

Therefore, $z \mapsto\left(H_{g}(\theta)-z\right)^{-1}$ is analytic in the rectangular domain $\mathcal{I}_{j}(\delta / 2)+$ $i\left[-S, g^{2}\right]$, and by Cauchy's integral formula, Eqn. (III.17) can be written as

$$
\left\langle\psi_{j} \mid \exp \left[-i t H_{g}\right] F_{j}\left(H_{g}\right) \psi_{j}\right\rangle=A_{+}-A_{-}-A_{\|},
$$

where

$$
\begin{gathered}
A_{ \pm}:=\int_{\delta / 4}^{\delta / 2} \frac{-F^{\prime}(\omega / \delta)}{4 i \pi \delta} \int_{0}^{S} \exp \left[-i t\left(E_{j} \pm \omega-i s\right)\right] \\
\left\{\left\langle\psi_{j}(\bar{\theta}) \mid\left(H_{g}(\theta)-E_{j} \mp \omega+i s\right)^{-1} \psi_{j}(\theta)\right\rangle\right. \\
\left.\quad-\left\langle\psi_{j}(\theta) \mid\left(H_{g}(\bar{\theta})-E_{j} \mp \omega+i s\right)^{-1} \psi_{j}(\bar{\theta})\right\rangle\right\} d s d \omega \\
A_{\|}:=\int_{\delta / 4}^{\delta / 2} \frac{-F^{\prime}(\omega / \delta)}{4 i \pi \delta} \int_{\mathcal{I}_{j}(\omega)} \exp [-i t(\lambda-i S)] \\
\\
\quad\left\{\left\langle\psi_{j}(\bar{\theta}) \mid\left(H_{g}(\theta)-\lambda+i S\right)^{-1} \psi_{j}(\theta)\right\rangle\right. \\
\left.-\left\langle\psi_{j}(\theta) \mid\left(H_{g}(\bar{\theta})-\lambda+i S\right)^{-1} \psi_{j}(\bar{\theta})\right\rangle\right\} d \lambda d \omega
\end{gathered}
$$

We first estimate $A_{\|}$. Since $P_{e l, j}$ and $e^{-H_{f} / g^{2}}$ are dilatation analytic and $\|\eta(\theta)\| \leq B_{\eta}$, we have for all $|\theta| \leq \theta_{0}$ that $\left\|\psi_{j}(\bar{\theta})\right\| \cdot\left\|\psi_{j}(\theta)\right\| \leq C B_{\eta}^{2}$, for some constant $C$ depending on $\theta_{0}$. Inserting this and (III.23) into the definition of 
$A_{\|}$, we obtain that

$$
\begin{gathered}
\left|A_{\|}\right|=\mid \int_{\delta / 4}^{\delta / 2} \frac{F^{\prime}(\omega / \delta)}{2 \pi \delta} \int_{\mathcal{I}_{j}(\omega)} \exp [-i t(\lambda-i S)] \\
\left\{\left\langle\psi_{j}(\bar{\theta}) \mid\left(H_{g}(\theta)-\lambda+i S\right)^{-1} \psi_{j}(\theta)\right\rangle\right. \\
\left.\quad-\left\langle\psi_{j}(\theta) \mid\left(H_{g}(\bar{\theta})-\lambda+i S\right)^{-1} \psi_{j}(\bar{\theta})\right\rangle\right\} d \lambda d \omega \mid \\
\leq C B_{\eta}^{2} e^{-t S} \max _{e \in \sigma\left(Z_{j}^{\mathrm{d}}(\theta)\right)}\left\{\int_{\mathcal{I}_{j}(\delta / 2)}\left[\left(E_{j}+g^{2} e-\lambda\right)^{2}+\left(C_{S} g^{2+\varepsilon} / 4\right)^{2}\right]^{-1 / 2} d \lambda\right\} \\
\leq C^{\prime} B_{\eta}^{2} \ln (1 / g) \exp \left[-t\left(g^{2} \Gamma_{j}-C_{S} g^{2+\varepsilon}\right)\right],
\end{gathered}
$$

for some constants $C, C^{\prime} \geq 0$ which depend on $\theta_{0}, \delta$, and $n_{j}$. Note that $H_{g}(\bar{\theta})-\lambda+i S$ is bounded invertible because the spectrum of $H_{g}(\bar{\theta})=H_{g}(\theta)^{*}$ lies in the upper half plane.

Second, we estimate $A_{+}$. We omit the similar estimate on $A_{-}$. We use the fact that, for any $N \in \mathbb{N}$ and $t>0$,

$$
\exp \left[-i t\left(E_{j}+\omega-i s\right)\right]=(-i t)^{-N} \frac{d^{N}}{d \omega^{N}}\left(\exp \left[-i t\left(E_{j}+\omega-i s\right)\right]\right) .
$$

Thus, an integration by parts yields

$$
\begin{aligned}
A_{+}= & \frac{i^{-N}}{2 \pi i \delta t^{N}} \int_{0}^{S} \int_{\delta / 4}^{\delta / 2} e^{-i t\left(E_{j}+\omega-i s\right)} \\
& \frac{d^{N}}{d \omega^{N}}\left(F ^ { \prime } ( \omega / \delta ) \left\{\left\langle\psi_{j}(\bar{\theta}) \mid\left(H_{g}(\theta)-E_{j}-\omega+i s\right)^{-1} \psi_{j}(\theta)\right\rangle\right.\right. \\
& \left.\left.-\left\langle\psi_{j}(\theta) \mid\left(H_{g}(\bar{\theta})-E_{j}-\omega+i s\right)^{-1} \psi_{j}(\bar{\theta})\right\rangle\right\}\right) d s d \omega \\
= & \frac{i^{-N}}{2 \pi i \delta t^{N}} \int_{0}^{S} \int_{\delta / 4}^{\delta / 2} e^{-i t\left(E_{j}+\omega-i s\right)} \sum_{k=0}^{N} \frac{N !}{(N-k) !} \delta^{-N+k} F^{(N-k+1)}(\omega / \delta) \\
& \left\{\left\langle\psi_{j}(\bar{\theta}) \mid\left(H_{g}(\theta)-E_{j}-\omega+i s\right)^{-k-1} \psi_{j}(\theta)\right\rangle\right. \\
& \left.\quad\left\langle\psi_{j}(\theta) \mid\left(H_{g}(\bar{\theta})-E_{j}-\omega+i s\right)^{-k-1} \psi_{j}(\bar{\theta})\right\rangle\right\} d s d \omega .
\end{aligned}
$$

Since all derivatives of $F$ are bounded and $S \leq g^{2} \Gamma_{j}$, there exists a constant $C_{N} \geq 0$ such that

$$
\begin{aligned}
\left|A_{+}\right| \leq \frac{C_{N} g^{2}}{t^{N}} \sup \{\mid & \left\langle\psi_{j}(\bar{\theta}) \mid\left(H_{g}(\theta)-E_{j}-\omega+i s\right)^{-k-1} \psi_{j}(\theta)\right\rangle \\
& -\left\langle\psi_{j}(\theta) \mid\left(H_{g}(\bar{\theta})-E_{j}-\omega+i s\right)^{-k-1} \psi_{j}(\bar{\theta})\right\rangle \mid \\
& \left.\mid 0 \leq k \leq N, \quad \frac{\delta}{4} \leq \omega \leq \frac{\delta}{2}, 0 \leq s \leq S\right\} .
\end{aligned}
$$


Thus, Theorem III.3 follows if we can find a constant $C>0$ such that

$$
\left|\left\langle\psi_{j}(\bar{\theta}) \mid R_{g}(\theta)^{k+1} \psi_{j}(\theta)\right\rangle-\left\langle\psi_{j}(\theta) \mid R_{g}(\bar{\theta})^{k+1} \psi_{j}(\bar{\theta})\right\rangle\right| \leq C B_{\eta}^{2} g^{2},
$$

for all $k \in\{0,1, \ldots, N\}, \omega \in[\delta / 4, \delta / 2]$, and $s \in[0, S]$, where we denote

$$
R_{g}(\theta):=\left(H_{g}(\theta)-E_{j}-\omega+i s\right)^{-1} \text {. }
$$

To this end we introduce an unperturbed resolvent,

$$
Q_{0}(\theta):=\left(H_{0}(\theta)-E_{j}-\omega-i g^{2}\right)^{-1},
$$

and we observe that, for $g /|\theta|$ sufficiently small,

$$
\begin{array}{r}
\left\|R_{g}(\theta)\right\|, \quad\left\|Q_{0}(\theta)\right\| \leq \quad C|\theta|^{-1}, \\
\left\|W_{g}(\theta) R_{g}(\theta)\right\|, \quad\left\|W_{g}(\theta) Q_{0}(\theta)\right\| \leq C g|\theta|^{-1},
\end{array}
$$

and some constant $C>0$, which is uniform in $\omega \in[\delta / 4, \delta / 2]$ and $s \in[0, S]$. Using the second resolvent equation, we obtain that

$$
\begin{aligned}
R_{g}(\theta)= & Q_{0}(\theta)-Q_{0}(\theta)\left[W_{g}(\theta)+i s+i g^{2}\right] Q_{0}(\theta) \\
& +Q_{0}(\theta)\left[W_{g}(\theta)+i s+i g^{2}\right] R_{g}(\theta)\left[W_{g}(\theta)+i s+i g^{2}\right] Q_{0}(\theta)
\end{aligned}
$$

We expand $R_{g}(\theta)^{k+1}$ by means of (III.36),

$$
R_{g}(\theta)^{k+1}=Q_{0}(\theta)^{k+1}-\sum_{\nu=1}^{k+1} Q_{0}(\theta)^{\nu} W_{g}(\theta) Q_{0}(\theta)^{k+2-\nu}+\text { Rem }
$$

and (III.34)-(III.35) show that there is a constant $C \geq 0$, depending on $k$ and $\theta$, such that

$$
\|\operatorname{Rem}\| \leq C g^{2}
$$

Similarly, we find that

$$
\begin{gathered}
R_{g}(\bar{\theta})^{k+1}=Q_{0}^{*}(\theta)^{k+1}-\sum_{\nu=1}^{k+1} Q_{0}^{*}(\theta)^{\nu} W_{g}(\bar{\theta}) Q_{0}^{*}(\theta)^{k+2-\nu}+\operatorname{Rem}^{\prime} \\
\left\|\operatorname{Rem}^{\prime}\right\| \leq C g^{2}
\end{gathered}
$$


(note that $Q_{0}^{*}(\theta) \neq Q_{0}(\bar{\theta})$ ). Inserting the two identities (III.37) and (III.39) into (III.31) and using (III.38) and (III.40), we observe that it suffices to prove that there is a constant $C \geq 0$ such that

$$
\begin{aligned}
&\left|\operatorname{Im}\left\{\left\langle\psi_{j}(\bar{\theta}) \mid Q_{0}(\theta)^{k+1} \psi_{j}(\theta)\right\rangle\right\}\right| \leq C B_{\eta}^{2} g^{2}, \\
&\left|\left\langle\psi_{j}(\bar{\theta}) \mid Q_{0}(\theta)^{\nu} W_{g}(\theta) Q_{0}(\theta)^{k+2-\nu} \psi_{j}(\theta)\right\rangle\right| \leq C B_{\eta}^{2} g^{2},
\end{aligned}
$$

for all $k \in\{0,1, \ldots, N\}, \nu \in\{1,2, \ldots, k+1\}$, and $\omega \in[\delta / 4, \delta / 2]$. We remark that (III.41) and (III.42) hold trivially for $\psi_{j}=\varphi_{j} \otimes \Omega$, where $\varphi_{j}$ is an normalized eigenvector of $H_{e l}$ corresponding to the eigenvalue $E_{j}$.

To prove (III.42), we observe that, for any two vectors $\psi \in \mathcal{D}\left(H_{f}^{m / 2}\right)$, $\varphi \in \mathcal{D}\left(H_{f}^{n / 2}\right)$, we have

$$
\begin{aligned}
& \left|\left\langle\psi \mid P_{e l, j}(\theta) W_{g}(\theta) P_{e l, j}(\theta) \varphi\right\rangle\right| \\
& \leq \sum_{m+n=1}^{2} g^{m+n} \int\left\|P_{e l, j}(\theta) w_{m, n}\left(\xi^{(m)}, \tilde{\xi}^{(n)} ; \theta\right) P_{e l, j}(\theta)\right\| \\
& \quad\left\|a\left(\xi^{(m)}\right) \psi\right\|\left\|a\left(\tilde{\xi}^{(n)}\right) \varphi\right\| d \xi^{(m)} d \tilde{\xi}^{(n)} \\
& \leq C \sum_{m+n=1}^{2} g^{m+n}\left(\int \frac{J(k)^{2} d^{3} k}{\omega(k)}\right)^{(m+n) / 2}\left\langle\psi \mid H_{f}^{m} \psi\right\rangle\left\langle\varphi \mid H_{f}^{n} \varphi\right\rangle \\
& \leq C^{\prime} \sum_{m+n=1}^{2}\left(\Lambda_{-1} g\right)^{m+n}\left\|H_{f}^{m / 2} \psi\right\|\left\|H_{f}^{n / 2} \varphi\right\|,
\end{aligned}
$$

by Schwarz' inequality. Here we abbreviate the summation $\sum_{\lambda_{1}=1}^{2} \cdots \sum_{\lambda_{m}=1}^{2}$ $\int d^{3} k_{1} \cdots d^{3} k_{m}$ by $d \xi^{(m)}$, and $\xi^{(m)}:=\left(k_{1}, \lambda_{1}, \ldots, k_{m}, \lambda_{m}\right)$. Thus we have

$$
\begin{aligned}
& \left|\left\langle\psi_{j}(\bar{\theta}) \mid Q_{0}(\theta)^{\nu} W_{g}(\theta) Q_{0}(\theta)^{k+2-\nu} \psi_{j}(\theta)\right\rangle\right| \\
& \quad=\left|\left\langle\left(Q_{0}(\theta)^{*}\right)^{\nu} \psi_{j}(\bar{\theta}) \mid P_{e l, j}(\theta) W_{g}(\theta) P_{e l, j}(\theta) Q_{0}(\theta)^{k+2-\nu} \psi_{j}(\theta)\right\rangle\right| \\
& \leq C \max _{m+n=1,2}\left\{g^{m+n}\left\|H_{f}^{m / 2}\left(Q_{0}(\theta)^{*}\right)^{\nu} \psi_{j}(\bar{\theta})\right\|\left\|H_{f}^{n / 2} Q_{0}(\theta)^{k+2-\nu} \psi_{j}(\theta)\right\|\right\},
\end{aligned}
$$

Next, we observe that

$$
\left(Q_{0}(\theta)^{*}\right)^{\nu} \psi_{j}(\bar{\theta})=\left(e^{-\bar{\theta}} H_{f}-\omega+i g^{2}\right)^{-\nu} \exp \left[-g^{-2} e^{-\bar{\theta}} H_{f}\right] P_{e l, j}(\bar{\theta}) \eta(\bar{\theta})
$$


and hence, for suitable constants $C, C^{\prime}, C^{\prime \prime} \geq 0$,

$$
\begin{aligned}
& \left\|H_{f}^{m / 2}\left(Q_{0}(\theta)^{*}\right)^{\nu} \psi_{j}(\bar{\theta})\right\| \\
& \leq C B_{\eta} \sup _{r \geq 0}\left|r^{m / 2}\left(e^{i \vartheta} r-\omega+i g^{2}\right)^{-\nu} \exp \left[-g^{-2} e^{i \vartheta} r\right]\right| \\
& \leq C^{\prime} B_{\eta} \vartheta^{-1} \sup _{r \geq 0}\left\{r^{m / 2} \exp \left[-g^{-2} e^{\vartheta} r\right]\right\} \leq C^{\prime \prime} B_{\eta} \vartheta^{-1}\left(g^{2} e^{\vartheta}\right)^{m / 2} .
\end{aligned}
$$

Inserting this and a similar estimate for $\left\|H_{f}^{n / 2} Q_{0}(\theta)^{k+2-\nu} \psi_{j}(\theta)\right\|$ into (III.44), we obtain that

$$
\begin{aligned}
\left|\left\langle\psi_{j}(\bar{\theta}) \mid Q_{0}(\theta)^{\nu} W_{g}(\theta) Q_{0}(\theta)^{k+2-\nu} \psi_{j}(\theta)\right\rangle\right| & \leq|\theta|^{-2} B_{\eta}^{2} \max _{m+n=1,2}\left\{\left(C g^{2}\right)^{m+n}\right\} \\
& =C|\theta|^{-2} B_{\eta}^{2} g^{2},
\end{aligned}
$$

for some constant $C \geq 0$ and $g>0$ sufficiently small. This proves (III.42).

Finally, we establish (III.41) by using the fact that we may analytically continue in $\theta$, since the spectral parameter $E_{j}+\omega+i g^{2}$ in $Q_{0}(\theta)$ is in the upper half-plane. Thus

$$
\begin{aligned}
\left\langle\psi_{j}(\bar{\theta}) \mid Q_{0}(\theta)^{k+1} \psi_{j}(\theta)\right\rangle & =\left\langle\psi_{j}(\bar{\theta}) \mid\left(e^{-\bar{\theta}} H_{f}-\omega-i g^{2}\right)^{-k-1} \psi_{j}(\theta)\right\rangle \\
& =\left\langle\psi_{j}(0) \mid\left(H_{f}-\omega-i g^{2}\right)^{-k-1} \psi_{j}(0)\right\rangle \\
& =\left\langle P_{e l, j} \eta \mid e^{-2 H_{f} / g^{2}}\left(H_{f}-\omega-i g^{2}\right)^{-k-1} P_{e l, j} \eta\right\rangle .
\end{aligned}
$$

Therefore,

$$
\left|\operatorname{Im}\left\langle\psi_{j}(\bar{\theta}) \mid Q_{0}(\theta)^{k+1} \psi_{j}(\theta)\right\rangle\right| \leq \sup _{r \geq 0}\left\{e^{-2 r / g^{2}}\left|\operatorname{Im}\left\{\left(r-\omega-i g^{2}\right)^{-k-1}\right\}\right|\right\} .
$$

Now, we use that $\omega \geq \delta / 4$. If $r \leq \omega / 2$ then $\omega-r \geq \delta / 8$ and thus

$$
\left|\arg \left(r-\omega-i g^{2}\right)\right| \leq \frac{\left|\operatorname{Im}\left(r-\omega-i g^{2}\right)\right|}{\left|\operatorname{Re}\left(r-\omega-i g^{2}\right)\right|} \leq \frac{8 g^{2}}{\delta} .
$$

Hence, for $r \leq \omega / 2$,

$$
\exp \left[-2 g^{-2} r\right]\left|\operatorname{Im}\left\{\left(r-\omega-i g^{2}\right)^{-k-1}\right\}\right| \leq(k+1)(8 / \delta)^{k+2} g^{2} .
$$

We point out that only for the derivation of (III.51) we need to estimate the imaginary part of a matrix element rather than its magnitude. It remains to consider the case $r \geq \omega / 2 \geq \delta / 8$. We estimate as follows,

$$
\exp \left[-2 g^{-2} r\right]\left|\operatorname{Im}\left\{\left(r-\omega-i g^{2}\right)^{-k-1}\right\}\right| \leq g^{-2 k-2} \exp \left[-\delta g^{-2} / 8\right] \leq C g^{2},
$$


for some constant $C \geq 0$. Inserting (III.51), (III.52) into (III.49), we obtain (III.41) which, together with (III.42), finishes the proof.

\section{III.2 Resolvent Norm Estimates and the Proof of Theo- rem III.1}

The purpose of this subsection is to prove Theorem III.1, i.e., that $H_{g}(\theta)-z$ is invertible for $z \in \mathcal{A}_{j}$, where $\mathcal{A}_{j}$ is defined in (III.12), and its inverse obeys the norm estimate

$$
\left\|\left(H_{g}(\theta)-z\right)^{-1}\right\| \leq C \operatorname{dist}\left\{z, \mathcal{R}_{j}\right\}
$$

for some $C>0$ and with $\mathcal{R}_{j}$ as defined in (III.10). Before we turn to the actual proof, we outline our strategy. We distinguish the spectral parameters $z$ close to the eigenvalue $E_{j}$ of $H_{e l}(\theta)$ from those which are away from $E_{j}$ by a certain minimal distance, $\rho_{0} / 2$, where

$$
\rho_{0}:=g^{2-2 \varepsilon},
$$

and $0<\varepsilon<1 / 3$ is arbitrary but fixed. Note that, given any $\delta, \vartheta, c>0$, we have

$$
\rho_{0} \leq \delta \sin (\vartheta / 2) \quad \text { and } \quad \rho_{0} \geq c g^{2-\varepsilon},
$$

provided $g \geq 0$ is sufficiently small.

For $z \in \mathcal{A}_{j} \backslash D\left(E_{j}, \rho_{0} / 2\right)$, a Neumann series expansion shows the invertibility of $H_{g}(\theta)-z$ and proves (III.53) for that region. This is proven in Lemma III.12 below.

For $z \in D\left(E_{j}, \rho / 2\right)$, the situation is more delicate, and we contruct the inverse of $H_{g}(\theta)-z$ by means of the Feshbach map discussed in detail in $[5,6]$. For this construction, we specify a partition of unity given by the (non-orthogonal) projections

$$
P(\theta):=P_{e l, j}(\theta) \otimes \chi_{H_{f}<\rho_{0}} \text { and } \bar{P}(\theta):=\mathbf{1}-P(\theta),
$$

where $P_{e l, j}(\theta):=U_{\theta} P_{e l, j} U_{\theta}^{-1}$ and $P_{e l, j}$ is the (orthogonal) projection onto the eigenspace of $H_{e l}$ corresponding to the eigenvalue $E_{j}$. In Lemma III.9 below we prove that

$$
H_{g}(\theta)_{\bar{P}(\theta)}-z \text { is invertible on } \operatorname{Ran}\{\bar{P}(\theta)\}
$$


where we denote $A_{P}:=P A P$. This property and some further relative bounds of more technical nature stated in Lemma III.10, choosing $\beta:=$ $2 \varepsilon(1-\varepsilon)^{-1} \in(0,1)$, insure the existence of the Feshbach operator defined by

$$
\begin{aligned}
& \mathcal{F}_{P(\theta)}:=\mathcal{F}_{P(\theta)}\left(H_{g}(\theta)-z\right) \\
& :=H_{g}(\theta)_{P(\theta)}-z P(\theta)-P(\theta) W_{g} \bar{P}(\theta)\left(H_{g}(\theta)_{\bar{P}(\theta)}-z\right)^{-1} \bar{P}(\theta) W_{g} P(\theta) .
\end{aligned}
$$

Moreover, the following identity holds,

$$
\begin{gathered}
\left(H_{g}(\theta)-z\right)^{-1}=\left[P(\theta)-\bar{P}(\theta)\left(H_{g}(\theta)_{\bar{P}(\theta)}-z\right)^{-1} W_{g} P(\theta)\right] \\
\times \mathcal{F}_{P(\theta)}^{-1}\left[P(\theta)-P(\theta) W_{g} \bar{P}(\theta)\left(H_{g}(\theta)_{\bar{P}(\theta)}-z\right)^{-1}\right] \\
+\bar{P}(\theta)\left(H_{g}(\theta)_{\bar{P}(\theta)}-z\right)^{-1} \bar{P}(\theta)
\end{gathered}
$$

Thus using bounds collected in Lemma III.10 below, we obtain that, for $z \in D\left(E_{j}, \rho_{0} / 2\right)$,

$$
\left\|\left(H_{g}(\theta)-z\right)^{-1}\right\|=\left(1+\mathcal{O}\left(g \rho_{0}^{-1} \vartheta^{-1}\right)\right)\left\|\mathcal{F}_{P(\theta)}^{-1}\right\|+\mathcal{O}(1) .
$$

Next, a careful analysis of the Feshbach operator in Lemma III.11 yields that

$$
\left\|\mathcal{F}_{P(\theta)}-\left(E_{j}+g^{2} Z_{j}^{\mathrm{d}}(\theta)+g^{2} Z_{j}^{\text {od }}(\theta)+e^{-\theta} H_{f}\right) P(\theta)\right\| \leq \mathcal{O}\left(g^{2+\varepsilon}\right),
$$

for any $0<\varepsilon<1 / 3$, which immediately implies (III.53).

\section{III.2.1 Estimates on the Dilated Atomic Hamiltonian}

In this subsection, we start to implement the strategy of the proof of Theorem III.1 outlined above. To begin with, we recall some definitions and notation. The pure point spectrum of $H_{e l}$ is given by the set $\left\{E_{0}, E_{1}, \ldots, E_{j}, \ldots\right\}$ contained in $(-\infty, \Sigma)$, and its essential spectrum is contained in $[\Sigma, \infty)$. We assume that $\Sigma \leq 0$ and, we denote $R_{i}:=\operatorname{dist}\left(E_{i}, \sigma\left(H_{e l}\right) \backslash\left\{E_{i}\right\}\right)>0$. Fixing $j \geq 1-$ and we shall keep $j \geq 1$ fixed henceforth - we set $\delta:=R_{j}$. So denoting $H_{e l}(\theta):=U_{\theta} H_{e l} U_{\theta}^{-1}$, we can construct the projection $P_{e l, i}(\theta)$ onto the eigenspace of $H_{e l}(\theta)$ corresponding to the eigenvalue $E_{i}$ by using the Dunford integral,

$$
P_{e l, i}(\theta)=\frac{i}{2 \pi} \int_{\left|z-E_{i}\right|=R_{i} / 2} \frac{d z}{H_{e l}(\theta)-z},
$$


Next, we define a finite-rank projection $P_{d i s c}(\theta)$ by

$$
P_{d i s c}(\theta):=\sum_{i: E_{i} \leq \Sigma-\mu} P_{e l, i}(\theta),
$$

where $\mu$ is some fixed, strictly positive number. Note that

$$
\left\|P_{\text {disc }}(\theta)-P_{\text {disc }}(0)\right\| \leq C b|\theta|,
$$

thanks to the relative bound (I.55),

$$
\left\|\Delta H_{e l}(\theta)\left(H_{e l}+i\right)^{-1}\right\| \leq b|\theta|,
$$

where $\Delta H_{e l}(\theta):=H_{e l}(\theta)-H_{e l}$.

Our first result is the following bound.

Lemma III.4. Let $z \in \mathbb{C}$ with $\operatorname{Re}\{z\}<\Sigma-\mu$. Then, for $|\theta|(1+(\Sigma-\mu-$ $\left.\operatorname{Re}\{z\})^{-1}\right)$ sufficiently small, $H_{e l}(\theta)-z$ is invertible on $\operatorname{Ran}\left\{\bar{P}_{\text {disc }}(\theta)\right\}$ and

$$
\left\|\left(H_{e l}(\theta)-z\right)^{-1} \bar{P}_{d i s c}(\theta)\right\| \leq \frac{2}{\Sigma-\mu-\operatorname{Re}\{z\}} .
$$

Proof: We first observe that $Q:=\bar{P}_{d i s c}(0) H_{e l}(0)-z$ is globally invertible on $\mathcal{H}_{e l}$, and since $\Sigma \leq 0$ we have

$$
\left\|Q^{-1}\right\| \leq \max \left\{|\Sigma-\mu-z|^{-1},|-z|^{-1}\right\} \leq(\Sigma-\mu-\operatorname{Re}\{z\})^{-1} .
$$

Similarly, we obtain

$$
\begin{aligned}
\left\|\left(H_{e l}(0)+i\right) Q^{-1}\right\| & =\max \left\{\sup _{r \geq 0}\left|\frac{r+\Sigma-\mu+i}{r+\Sigma-\mu-z}\right|, \sup _{E_{0} \leq r \leq \Sigma-\mu}\left|\frac{r+i}{z}\right|\right\} \\
& \leq C_{1}\left(1+(\Sigma-\mu-\operatorname{Re}\{z\})^{-1}\right),
\end{aligned}
$$

for some constant $C_{1} \geq 0$. Inserting this and (III.64)-(III.65), we obtain

$$
\begin{aligned}
& \|\left(\bar{P}_{d i s c}(0)\right.\left.H_{e l}(0)-\bar{P}_{d i s c}(\theta) H_{e l}(\theta)\right) Q^{-1} \| \\
& \leq C_{2}|\theta|\left(1+(\Sigma-\mu-\operatorname{Re}\{z\})^{-1}\right),
\end{aligned}
$$

for some constant $C_{2} \geq 0$. Thus a Neumann series expansion yields

$$
\begin{aligned}
& \left\|\left(H_{e l}(\theta)-z\right)^{-1} \bar{P}_{d i s c}(\theta)\right\|=\left\|\left(\bar{P}_{d i s c}(\theta) H_{e l}(\theta)-z\right)^{-1} \bar{P}_{d i s c}(\theta)\right\| \\
& \quad \leq\left\|Q^{-1} \sum_{n=0}^{\infty}\left(\left(\bar{P}_{d i s c}(0) H_{e l}(0)-\bar{P}_{d i s c}(\theta) H_{e l}(\theta)\right) Q^{-1}\right)^{n} \bar{P}_{d i s c}(\theta)\right\| \\
& \quad \leq \frac{1}{\Sigma-\mu-\operatorname{Re}\{z\}} \sum_{n=0}^{\infty}\left(C_{2}|\theta|+\frac{C_{2}|\theta|}{\Sigma-\mu-\operatorname{Re}\{z\}}\right)^{n} \leq \frac{2}{\Sigma-\mu-\operatorname{Re}\{z\}},
\end{aligned}
$$


for $|\theta|\left(1+(\Sigma-\mu-\operatorname{Re}\{z\})^{-1}\right) \leq\left(2 C_{2}\right)^{-1}$.

Next, we extend Lemma III.4 to a global bound for the resolvent of $H_{e l}$.

Lemma III.5. Let $\rho>0$. For $\theta=i \vartheta$ and $\vartheta>0$ sufficiently small, there exists a constant $C \geq 0$ such that $H_{e l}(\theta)-E_{j}+e^{-\theta} \rho$ is invertible on $\mathcal{H}_{\text {el }}$ and

$$
\left\|\left(H_{e l}(\theta)-E_{j}+e^{-\theta} \rho\right)^{-1}\right\| \leq C(\vartheta \rho)^{-1} .
$$

Proof: We observe that $H_{e l}(\theta)$ commutes with $P_{d i s c}(\theta)=\sum_{i: E_{i} \leq \Sigma-\mu} P_{e l, i}(\theta)$ and that

$$
\left(H_{e l}(\theta)-E_{j}+e^{-\theta} \rho\right)^{-1} P_{d i s c}(\theta)=\sum_{i: E_{i} \leq \Sigma-\mu}\left(E_{i}-E_{j}+e^{-\theta} \rho\right)^{-1} P_{e l, i}(\theta) .
$$

Thus, for some constant $C^{\prime} \geq 0$,

$$
\begin{aligned}
& \left\|\left(H_{e l}(\theta)-E_{j}+e^{-\theta} \rho\right)^{-1} P_{d i s c}(\theta)\right\| \\
& \quad \leq \max _{i: E_{i} \leq \Sigma-\mu}\left\{\frac{C^{\prime}}{\rho \vartheta}\left\|P_{e l, i}(\theta)\right\|\right\} \cdot \#\left\{E_{i} \in \sigma\left(H_{e l}\right) \mid E_{i} \leq \Sigma-\mu\right\} .
\end{aligned}
$$

Using the integral representation $P_{e l, i}(\theta)=(2 \pi i)^{-1} \int_{\left|z-E_{i}\right|=R_{i} / 2}\left(z-H_{e l}(\theta)\right)^{-1} d z$ together with the relative bound (III.65), we obtain that $\left\|P_{e l, i}(\theta)\right\| \leq 1+$ $\mathcal{O}(|\theta|)$. Conversely, on $\operatorname{Ran}\left\{\bar{P}_{d i s c}(\theta)\right\}$ we apply Lemma III.4 and obtain

$$
\begin{aligned}
\left\|\left(H_{e l}(\theta)-E_{j}+e^{-\theta} \rho\right)^{-1} \bar{P}_{d i s c}(\theta)\right\| & \leq\left(\Sigma-\mu-E_{j}+\rho \cdot \operatorname{Re}\left\{e^{-\theta}\right\}\right)^{-1} \\
& \leq 2(\cos (\vartheta) \rho)^{-1}
\end{aligned}
$$

Lemma III.6. Let $\theta= \pm i \vartheta, 0<\vartheta<\theta_{0}$ and $0<\rho_{0} \leq(\delta / 3) \sin \vartheta$. There exists a constant $C \geq 0$ such that, for all $z \in D\left(E_{j}, \rho_{0} / 2\right)$ and all $r \geq 0$,

$$
\left\|\left(H_{e l}(\theta)-E_{j}+e^{-\theta}\left(\rho_{0}+r\right)\right)\left(\frac{\bar{P}_{e l, j}(\theta)}{H_{e l}(\theta)-z+e^{-\theta} r}\right)\right\| \leq C .
$$


Proof: Using $\mathbf{1}=P_{d i s c}(\theta)+\bar{P}_{d i s c}(\theta)$ as in the proof of Lemma III.5, we obtain that

$$
\begin{aligned}
& \left\|X \bar{P}_{e l, j}(\theta)\right\| \\
& \leq\left\|X \bar{P}_{d i s c}(\theta)\right\|+\sum_{i: E_{i} \leq \Sigma-\varepsilon, i \neq j}\left|\frac{E_{i}-E_{j}+e^{-\theta}\left(\rho_{0}+r\right)}{E_{i}-z+e^{-\theta} r}\right|\left\|P_{e l, j}(\theta)\right\| \\
& \leq\left\|X \bar{P}_{d i s c}(\theta)\right\|+ \\
& \quad C \sup \left\{\left|\frac{t+e^{-\theta}\left(\rho_{0}+r\right)}{t+\zeta+e^{-\theta} r}\right| \mid t \in \mathbb{R} \backslash(-\delta, \delta), \quad \zeta \in D\left(0, \rho_{0} / 2\right)\right\},
\end{aligned}
$$

where we denote

$$
X:=\left(H_{e l}(\theta)-E_{j}+e^{-\theta}\left(\rho_{0}+r\right)\right)\left(H_{e l}(\theta)-z+e^{-\theta} r\right)^{-1} .
$$

We observe that minimization with respect to $r \geq 0$ yields

$$
\begin{aligned}
\left|\frac{t+\zeta+e^{-\theta} r}{t+e^{-\theta}\left(\rho_{0}+r\right)}\right| & =\left|1-\frac{e^{-\theta} \rho_{0}-\zeta}{t+e^{-\theta}\left(\rho_{0}+r\right)}\right| \\
& \geq 1-\frac{3 \rho_{0}}{2}\left[\left(t+\left(\rho_{0}+r\right) \cos \vartheta\right)^{2}+\left(\rho_{0}+r\right)^{2} \sin ^{2} \vartheta\right]^{-1 / 2} \\
& \geq 1-\frac{3 \rho_{0}}{2}\left[(1-\cos \vartheta)\left(t^{2}+\left(\rho_{0}+r\right)^{3}\right)\right]^{-1 / 2} \geq \frac{1}{2},
\end{aligned}
$$

additionally taking into account that $\rho_{0} \leq(\delta / 3) \sin \vartheta$. Hence

$$
\left\|X \bar{P}_{e l, j}(\theta)\right\| \leq\left\|X \bar{P}_{d i s c}(\theta)\right\|+2 C .
$$

Next, we write

$$
X \bar{P}_{d i s c}(\theta)=\bar{P}_{d i s c}(\theta)+\left(e^{-\theta} \rho_{0}+z-E_{j}\right)\left(H_{e l}(\theta)-z+e^{-\theta} r\right)^{-1} \bar{P}_{d i s c}(\theta),
$$

and we obtain from Lemma III.4 that

$$
\left\|X \bar{P}_{d i s c}(\theta)\right\| \leq\left\|\bar{P}_{d i s c}(\theta)\right\|\left[1+\frac{3 \rho_{0}}{2}(\Sigma-\mu-\operatorname{Re}\{z\})^{-1}\right] \leq C,
$$

and hence we arrive at the claim. 


\section{III.2.2 Relative Bounds on the Interaction}

In this subsection we use the estimates on the dilated electron Hamiltonian derived in the previous subsection to obtain suitable relative bounds on the interaction. To this end, we recall Eqns. (I.37)-(I.45), and we introduce the operator

$$
B_{\theta}(\rho):=H_{0}(\theta)-E_{j}+e^{-\theta} \rho=H_{e l}(\theta)-E_{j}+e^{-\theta}\left(H_{f}+\rho\right) .
$$

We shall generally assume that $0<\vartheta \leq \theta_{0}$ and that $0<\rho_{0} \leq(\delta / 3) \sin \vartheta$. As before, we denote $H_{g}(\theta)_{\bar{P}(\theta)}:=\bar{P}(\theta) H_{g}(\theta) \bar{P}(\theta)$ and $H_{0}(\theta)_{\bar{P}(\theta)}:=H_{0}(\theta) \bar{P}(\theta)$. Finally, for a closed operator $A$ we denote $|A|:=\sqrt{A^{*} A}$. We start with a preparatory lemma.

Lemma III.7. For $\theta= \pm i \vartheta$ and $\vartheta \in\left(0, \theta_{0}\right)$ sufficiently small there exists a constant $C \geq 0$ such that

$$
\begin{aligned}
\left\|\left|B_{\theta}(\rho)\right|^{-1}\left(H_{f}+\omega\right)\right\| & \leq \frac{C}{\vartheta}\left(1+\frac{\omega}{\rho}\right), \\
\left\|\left|B_{\theta}(\rho)\right|^{-1}\left(H_{e l}(0)+i\right)\right\| & \leq \frac{C}{\vartheta}\left(1+\frac{1}{\rho}\right),
\end{aligned}
$$

for all $\rho>0$ and $\omega \geq 0$.

Proof: By the functional calculus and Lemma III.5 we have

$$
\begin{aligned}
\left\|\left|B_{\theta}(\rho)\right|^{-1}\left(H_{f}+\omega\right)\right\| & =\sup _{r \geq 0}\left\{\left\|\left(H_{e l}(\theta)-E_{j}+e^{-\theta}(r+\rho)\right)^{-1}\right\|(r+\omega)\right\} \\
& \leq C \sup _{r>0}\left\{\frac{r+\omega}{\vartheta(r+\rho)}\right\} \leq \frac{C}{\vartheta} \max \left\{1, \frac{\omega}{\rho}\right\}, \quad \text { (III.86) }
\end{aligned}
$$

which implies (III.84). To establish (III.85) we start with a similar observation, namely, that

$$
\left\|\left|B_{\theta}(\rho)\right|^{-1}\left(H_{e l}(0)+i\right)\right\|=\sup _{r \geq 0}\|Y\|_{\mathcal{H}_{e l}}
$$

where

$$
Y:=\left(H_{e l}(\theta)-E_{j}+e^{-\theta}(r+\rho)\right)^{-1}\left(H_{e l}(0)+i\right) .
$$

We observe the following identity,

$$
\begin{aligned}
Y= & \mathbf{1}-Y\left(H_{e l}(0)+i\right)^{-1} \Delta H_{e l}(\theta) \\
& +\left(i+E_{j}-e^{-\theta}(r+\rho)\right)\left(H_{e l}(\theta)-E_{j}+e^{-\theta}(r+\rho)\right)^{-1} .
\end{aligned}
$$


Solving for $Y$ and applying Lemma III.5, we obtain

$$
\|X(\theta)\| \leq \frac{1}{1-b|\theta|}\left(1+C\left|i+E_{j}-e^{-\theta} \rho\right|\left(1+\frac{1}{\vartheta \rho}\right)\right) \leq C\left(1+\frac{1}{\vartheta \rho}\right) .
$$

Now, we come to the main relative bound used in this section.

Lemma III.8. For $\theta, \theta_{1}, \theta_{2} \in\{ \pm i \vartheta\}, 0<\beta<1$, and $\vartheta \in\left(0, \theta_{0}\right)$ sufficiently small there exists a constant $C_{\beta} \geq 0$ such that

$$
\begin{aligned}
\left\|\left|B_{\theta_{1}}(\rho)\right|^{-1 / 2} W_{m, n}(\theta)\left|B_{\theta_{2}}(\rho)\right|^{-1 / 2}\right\| \leq C_{\beta}\left(1+\rho^{-1 / 2}\right) \\
\left\|\left|B_{\theta_{1}}(\rho)\right|^{-1 / 2} W_{m, n}(\theta) P(\theta)\right\| \leq C_{\beta}\left(1+\rho^{-1 / 2}\right)\left(\rho+\rho_{0}\right)^{1 / 2} \rho_{0}^{n \beta / 2}, \\
\left\|P(\theta) W_{m, n}(\theta)\left|B_{\theta_{2}}(\rho)\right|^{-1 / 2}\right\| \leq C_{\beta}\left(1+\rho^{-1 / 2}\right)\left(\rho+\rho_{0}\right)^{1 / 2} \rho_{0}^{m \beta / 2}, \\
\left\|P(\theta) W_{m, n}(\theta) P(\theta)\right\| \leq C_{\beta}\left(1+\rho^{-1 / 2}\right)\left(\rho+\rho_{0}\right) \rho_{0}^{(m+n) \beta / 2},
\end{aligned}
$$

for all $\rho>0$.

Proof: We first observe that $a_{\lambda}(\vec{k}) B_{\theta}(\rho)=B_{\theta}(\rho+\omega(k)) a_{\lambda}(\vec{k})$ and thus

$$
\begin{aligned}
& a_{\lambda}(\vec{k})\left|B_{\theta}(\rho)\right|^{2}=a_{\lambda}(\vec{k}) B_{\bar{\theta}}(\rho) B_{\theta}(\rho) \\
& \quad=B_{\bar{\theta}}(\rho+\omega(k)) B_{\theta}(\rho+\omega(k)) a_{\lambda}(\vec{k})=\left|B_{\theta}(\rho+\omega(k))\right|^{2} a_{\lambda}(\vec{k}) .
\end{aligned}
$$

Thus, functional calculus implies the Pull-Through Formulae

$$
\begin{aligned}
a_{\lambda}(\vec{k})\left|B_{\theta}(\rho)\right|^{-1 / 2} & =\left|B_{\theta}(\rho+\omega(k))\right|^{-1 / 2} a_{\lambda}(\vec{k}), \\
\left|B_{\theta}(\rho)\right|^{-1 / 2} a_{\lambda}^{*}(\vec{k}) & =a_{\lambda}^{*}(\vec{k})\left|B_{\theta}(\rho+\omega(k))\right|^{-1 / 2} .
\end{aligned}
$$

Using (III.96)-(III.97), we observe that, for any $\psi \in \mathcal{H}$,

$$
\begin{aligned}
& \left\|\left|B_{\theta_{1}}(\rho)\right|^{-1 / 2} W_{0,1}(\theta)\left|B_{\theta_{2}}(\rho)\right|^{-1 / 2} \psi\right\| \\
& =\left\|\sum_{\lambda=1}^{2} \int d^{3} k\left|B_{\theta_{1}}(\rho)\right|^{-1 / 2} w_{0,1}(\vec{k}, \lambda ; \theta)\left|B_{\theta_{2}}(\rho+\omega)\right|^{-1 / 2} a_{\lambda}(\vec{k}) \psi\right\| \\
& =\sup _{\lambda=1,2}\left\{\int\left\|\left|B_{\theta_{1}}(\rho)\right|^{-1 / 2} w_{0,1}(\vec{k}, \lambda ; \theta)\left|B_{\theta_{2}}(\rho+\omega)\right|^{-1 / 2}\left(H_{f}+\omega\right)^{1 / 2}\right\|^{2} \frac{d^{3} k}{\omega}\right\}^{1 / 2} \\
& \times\left\{\sum_{\lambda=1}^{2} \int\left\|\left(H_{f}+\omega\right)^{-1 / 2} a_{\lambda}(\vec{k}) \psi\right\|^{2} \omega d^{3} k\right\}^{1 / 2},
\end{aligned}
$$


where here and henceforth we denote $\omega:=\omega(k)$ and $\omega^{\prime}:=\omega\left(k^{\prime}\right)$. Note that there is an additional constraint $\omega(k) \leq \rho_{0}$ in the integrals on the right side of (III.98) if we require that $\psi \in \operatorname{Ran} \chi_{H_{f}<\rho_{0}}$. The last factor in (III.98) equals

$$
\left\{\sum_{\lambda=1}^{2} \int\left\langle\psi \mid P_{\Omega}^{\perp} H_{f}^{-1 / 2} a_{\lambda}^{*}(\vec{k}) a_{\lambda}(\vec{k}) H_{f}^{-1 / 2} P_{\Omega}^{\perp} \psi\right\rangle \omega d^{3} k\right\}^{1 / 2} \leq\|\psi\| .
$$

Since furthermore $H_{f}+\omega$ commutes with and $B_{\theta_{1}}(\rho)$ and $w_{0,1}(\vec{k}, \lambda ; \theta)$, we may use (I.42) and Lemma III.7 to estimate

$$
\begin{aligned}
\| \mid & \left.B_{\theta_{1}}(\rho)\right|^{-1 / 2} w_{0,1}(\vec{k}, \lambda ; \theta)\left|B_{\theta_{2}}(\rho+\omega)\right|^{-1 / 2}\left(H_{f}+\omega\right)^{1 / 2} \| \\
\leq & \left\|\left|B_{\theta_{1}}(\rho)\right|^{-1 / 2}\left(H_{f}+\omega\right)^{1 / 2}\right\| \cdot\left\|w_{0,1}(\vec{k}, \lambda ; \theta)\left|H_{e l}(0)+i\right|^{-1 / 2}\right\| \\
& \cdot\left\|\left|H_{e l}(0)+i\right|^{1 / 2}\left|B_{\theta_{2}}(\rho+\omega)\right|^{-1 / 2}\left(H_{f}+\omega\right)^{1 / 2}\right\| \\
\leq & \frac{C}{\vartheta}\left(1+\frac{\omega}{\rho}\right)^{1 / 2}\left(1+\frac{1}{\rho+\omega}\right)^{1 / 2} J(k) \\
\leq & \frac{C}{\vartheta}\left(1+\frac{1}{\rho}\right)^{1 / 2}(1+\omega)^{1 / 2} J(k),
\end{aligned}
$$

for some constant $C \geq 0$. Inserting (III.100) and (III.99) into (III.98), we obtain that

$$
\begin{aligned}
& \left\|\left|B_{\theta_{1}}(\rho)\right|^{-1 / 2} W_{0,1}(\theta)\left|B_{\theta_{2}}(\rho)\right|^{-1 / 2}\right\|^{2} \\
& \leq \frac{C}{\vartheta^{2}}\left(1+\rho^{-1}\right) \int\left(1+\omega(k)^{-1}\right) J(k)^{2} d^{3} k \leq \frac{C\left(\Lambda_{0}+\Lambda_{-1}\right)}{\vartheta^{2}}\left(1+\rho^{-1}\right),
\end{aligned}
$$

for some constant $C \geq 0$. Similarly, by additionally requiring that $\psi \in$ $\operatorname{Ran} \chi_{H_{f}<\rho_{0}}$, we obtain that

$$
\begin{aligned}
\| & \left|B_{\theta_{1}}(\rho)\right|^{-1 / 2} W_{0,1}(\theta)\left|B_{\theta_{2}}(\rho)\right|^{-1 / 2} \chi_{H_{f}<\rho_{0}} \|^{2} \\
& \leq \frac{C}{\vartheta^{2}}\left(1+\rho^{-1}\right) \int_{\omega(k) \leq \rho_{0}}\left(1+\omega(k)^{-1}\right) J(k)^{2} d^{3} k \\
& \leq \frac{C \rho_{0}^{\beta}}{\vartheta^{2}}\left(1+\rho^{-1}\right) \int\left(\omega(k)^{-\beta}+\omega(k)^{-1-\beta}\right) J(k)^{2} d^{3} k \\
& \leq \frac{C\left(\Lambda_{-\beta}+\Lambda_{-1-\beta}\right) \rho_{0}^{\beta}}{\vartheta^{2}}\left(1+\rho^{-1}\right) .
\end{aligned}
$$

The estimate for $W_{1,0}(\theta)$ is similar. Next, we derive (III.91) in the case of 
$W_{0,2}(\theta)$. Picking $\psi \in \mathcal{H}$, we observe that

$$
\begin{aligned}
& \left\|\left|B_{\theta_{1}}(\rho)\right|^{-1 / 2} W_{0,2}(\theta)\left|B_{\theta_{2}}(\rho)\right|^{-1 / 2} \psi\right\| \\
& \leq \sup _{\lambda, \lambda^{\prime}=1,2}\left\{\int \|\left|B_{\theta_{1}}(\rho)\right|^{-1 / 2} w_{0,2}\left(\vec{k}, \vec{k}^{\prime}, \lambda, \lambda^{\prime} ; \theta\right)\right. \\
& \left.\quad \times\left. B_{\theta_{2}}\left(\rho+\omega+\omega^{\prime}\right)\right|^{-1 / 2}\left(H_{f}+\omega+\omega^{\prime}\right) \|^{2} \frac{d^{3} k d^{3} k^{\prime}}{\omega \omega^{\prime}}\right\}^{1 / 2} \\
& \quad \times\left\{\sum_{\lambda, \lambda^{\prime}=1}^{2} \int\left\|\left(H_{f}+\omega+\omega^{\prime}\right)^{-1} a_{\lambda^{\prime}}\left(\vec{k}^{\prime}\right) a_{\lambda}(\vec{k}) \psi\right\|^{2} \omega \omega^{\prime} d^{3} k\right\}^{1 / 2} .
\end{aligned}
$$

Again, we have the additional constraint $\omega(k) \leq \rho_{0}$ in the integrals on the right side of (III.103) if we require that $\psi \in \operatorname{Ran} \chi_{H_{f}<\rho_{0}}$. The last factor is bounded by $\|\psi\|$. Thus Eqn. (I.43) and Lemma III.7 imply that, for some constants $C \geq 0$,

$$
\begin{aligned}
& \left\|\left|B_{\theta_{1}}(\rho)\right|^{-1 / 2} W_{0,2}(\theta)\left|B_{\theta_{2}}(\rho)\right|^{-1 / 2}\right\|^{2} \\
& \leq \int\left\|\left|B_{\theta_{1}}(\rho)\right|^{-1 / 2}\left(H_{f}+\omega+\omega^{\prime}\right)^{1 / 2}\right\|^{2} \\
& \quad \cdot\left\|\left|B_{\theta_{2}}\left(\rho+\omega+\omega^{\prime}\right)\right|^{-1 / 2}\left(H_{f}+\omega+\omega^{\prime}\right)^{1 / 2}\right\|^{2} \frac{J(k)^{2} J\left(k^{\prime}\right)^{2} d^{3} k d^{3} k^{\prime}}{\omega \omega^{\prime}} \\
& \leq \frac{C}{\vartheta^{2}} \int\left(1+\frac{\omega+\omega^{\prime}}{\rho}\right)\left(1+\frac{\omega+\omega^{\prime}}{\rho+\omega+\omega^{\prime}}\right) \frac{J(k)^{2} J\left(k^{\prime}\right)^{2} d^{3} k d^{3} k^{\prime}}{\omega \omega^{\prime}} \\
& \leq \frac{C\left(\Lambda_{0}+\Lambda_{-1}\right)^{2}}{\vartheta^{2}}\left(1+\rho^{-1}\right)
\end{aligned}
$$

and

$$
\begin{aligned}
\left\|\left|B_{\theta_{1}}(\rho)\right|^{-1 / 2} W_{0,2}(\theta)\left|B_{\theta_{2}}(\rho)\right|^{-1 / 2} \chi_{H_{f}<\rho_{0}}\right\|^{2} \\
\leq \frac{C\left(\Lambda_{-\beta}+\Lambda_{-1-\beta}\right) \rho_{0}^{2 \beta}}{\vartheta^{2}}\left(1+\rho^{-1}\right) .
\end{aligned}
$$

Estimates similar to (III.103)-(III.104) establish (III.91) in the remaining cases, i.e., for $W_{1,1}(\theta)$ and $W_{2,0}(\theta)$. Finally, we observe that $P(\theta)=\chi_{H_{f}<\rho_{0}} P(\theta)$ and

$$
\left\|B_{\theta}(\rho) P(\theta)\right\|=\left\|\left(H_{f}+\rho\right) P(\theta)\right\|=\rho_{0}+\rho,
$$

which together with (III.102) and (III.105) yields (III.92)-(III.94). 


\section{III.2.3 Domain of the Feshbach Map}

In the following subsection we apply the relative bounds from Lemma III.8 to prove that, for $z$ sufficiently close to $E_{j}$, the Feshbach map with projection $P(\theta)$ is applicable to $H_{g}(\theta)-z$.

Lemma III.9. Let $\rho_{0}<(\delta / 3) \sin \vartheta$, and assume that $\vartheta \in\left(0, \theta_{0}\right)$ is sufficiently small. Then, for $g \rho_{0}^{-1 / 2}>0$ sufficiently small and for all $z \in$ $D\left(E_{j}, \rho_{0} / 2\right)$, the operator $H_{g}(\theta)_{\bar{P}(\theta)}-z$ is invertible on $\operatorname{Ran}\{\bar{P}(\theta)\}$, and

$$
\left\|\left(H_{g}(\theta)_{\bar{P}(\theta)}-z\right)^{-1} \bar{P}(\theta)\right\| \leq \frac{C}{\vartheta \rho_{0}},
$$

for some constant $C \geq 0$.

Proof: We construct $\left(H_{g}(\theta)_{\bar{P}(\theta)}-z\right)^{-1} \bar{P}(\theta)$ by a norm-convergent Neumann series,

$$
\left(H_{g}(\theta)_{\bar{P}(\theta)}-z\right)^{-1} \bar{P}(\theta)=\sum_{n=0}^{\infty}\left(\frac{\bar{P}(\theta)}{H_{0}(\theta)-z}\right)\left[-W_{g}(\theta)\left(\frac{\bar{P}(\theta)}{H_{0}(\theta)-z}\right)\right]^{n} .
$$

We estimate the norm of the term in $n^{\text {th }}$ order by means of Lemma III.8,

$$
\begin{aligned}
& \left\|\left(\frac{\bar{P}(\theta)}{H_{0}(\theta)-z}\right)\left[-W_{g}(\theta)\left(\frac{\bar{P}(\theta)}{H_{0}(\theta)-z}\right)\right]^{n}\right\| \\
& \leq\left\|\left|B_{\theta}\left(\rho_{0}\right)\right|^{1 / 2}\left(\frac{\bar{P}(\theta)}{H_{0}(\theta)-z}\right)\left|B_{\bar{\theta}}\left(\rho_{0}\right)\right|^{1 / 2}\right\|^{n+1} \\
& \quad\left\|\left|B_{\bar{\theta}}\left(\rho_{0}\right)\right|^{-1 / 2}\right\| \cdot\left\|\left|B_{\theta}\left(\rho_{0}\right)\right|^{-1 / 2}\right\| \cdot\left\|\left|B_{\bar{\theta}}\left(\rho_{0}\right)\right|^{-1 / 2} W_{g}(\theta)\left|B_{\theta}\left(\rho_{0}\right)\right|^{-1 / 2}\right\|^{n} \\
& \leq \frac{C}{\vartheta \rho_{0}}\left(C g \rho_{0}^{-1 / 2}\right)^{n} .
\end{aligned}
$$

This proves the convergence of the Neumann series (III.108) in norm.

Lemma III.9 is the main ingredient used to prove the existence of the Feshbach operator defined in (III.58)-(III.60).

Lemma III.10. Let $\rho_{0}<(\delta / 3) \sin \vartheta$, and assume that $\vartheta \in\left(0, \theta_{0}\right)$ and $g>0$ are sufficiently small. Then, for all $z \in D\left(E_{j}, \rho_{0} / 2\right)$, the Feshbach operator defined in (III.58) exists and obeys Eqn. (III.59). Moreover, for some 
constant $C \geq 0$, we have

$$
\left.\begin{array}{c}
\left\|P(\theta) W_{g}(\theta) \bar{P}(\theta)\left(H_{g}(\theta)_{\bar{P}(\theta)}-z\right)^{-1} \bar{P}(\theta)\right\|, \\
\left\|\bar{P}(\theta)\left(H_{g}(\theta)_{\bar{P}(\theta)}-z\right)^{-1} \bar{P}(\theta) W_{g}(\theta) P(\theta)\right\|
\end{array}\right\} \leq \frac{C g}{\vartheta \rho_{0}^{1 / 2}} .
$$

Proof: The proof of (III.110) is similar to the one for Lemma III.9. Then Lemma III.9 and (III.108) imply the existence of the Feshbach operator defined in (III.58) and that it obeys Eqn. (III.59) (see, e.g., [5, 6]).

We finally establish two lemmata which, inserted into (III.61) and (III.53), prove Theorem III.1.

Lemma III.11. Let $0<\varepsilon<1 / 3, \rho_{0}:=g^{2-2 \varepsilon}, 0<\beta<1$, and assume that $\vartheta \in\left(0, \theta_{0}\right)$ and $g>0$ are sufficiently small and such that $\rho_{0}<(\delta / 3) \sin \vartheta$. Then, for all $z \in D\left(E_{j}, \rho_{0} / 2\right)$,

$$
\begin{gathered}
\left\|\mathcal{F}_{P(\theta)}-\left(E_{j}+g^{2} Z_{j}^{\mathrm{d}}(\theta)+g^{2} Z_{j}^{\mathrm{od}}(\theta)+e^{-\theta} H_{f}\right) P(\theta)\right\| \\
\leq C\left(g^{2+\varepsilon}+g^{2+2 \beta(1-\varepsilon)}+g^{1+(1+\beta)(1-\varepsilon)}+g^{4-2 \varepsilon}\right),
\end{gathered}
$$

for some constant $C \geq 0$.

Proof: Recall from (III.58) and (III.7)-(III.8) that

$$
\begin{aligned}
& \mathcal{F}_{P(\theta)}:=\mathcal{F}_{P(\theta)}\left(H_{g}(\theta)-z\right) \\
& :=H_{g}(\theta)_{P(\theta)}-z P(\theta)-P(\theta) W_{g} \bar{P}(\theta)\left(H_{g}(\theta)_{\bar{P}(\theta)}-z\right)^{-1} \bar{P}(\theta) W_{g} P(\theta)
\end{aligned}
$$

and

$$
\begin{gathered}
Z_{j}^{\mathrm{od}}(\theta):=\sum_{\lambda=1,2} \int U_{\theta} P_{e l, j} w_{0,1}(\vec{k}, \lambda) P_{e l, j}^{\perp} \\
\left(H_{e l}-E_{j}+\omega(\vec{k})-i 0\right)^{-1} P_{e l, j}^{\perp} w_{1,0}(\vec{k}, \lambda) P_{e l, j} U_{\theta}^{-1} d^{3} k \\
Z_{j}^{\mathrm{d}}(\theta):=\sum_{\lambda=1,2} \int U_{\theta} P_{e l, j} w_{0,1}(\vec{k}, \lambda) P_{e l, j} w_{1,0}(\vec{k}, \lambda) P_{e l, j} U_{\theta}^{-1} \frac{d^{3} k}{\omega(\vec{k})},
\end{gathered}
$$


where $P_{e l, j}=P_{e l, j}(\theta=0)=\sum_{\ell=1}^{n_{j}}\left|\varphi_{j, \ell}\right\rangle\left\langle\varphi_{j, \ell}\right|$ is the orthogonal projection onto the eigenspace of $H_{e l}$ corresponding to the eigenvalue $E_{j}$. As in [5], we write the difference to be estimated as a sum of six error terms,

$$
\mathcal{F}_{P(\theta)}-\left(E_{j}+g^{2} Z_{j}^{\mathrm{d}}(\theta)+g^{2} Z_{j}^{\mathrm{od}}(\theta)+e^{-\theta} H_{f}\right) P(\theta)=\sum_{\mu=0}^{5} \operatorname{Rem}_{\mu},
$$

where (compare to [5, (IV.58), (IV.60), (IV.68), (IV.77), (IV.101), (IV.86), and (IV.87)])

$$
\begin{aligned}
& \operatorname{Rem}_{0}:=P(\theta) W_{g}(\theta) \bar{P}(\theta)[\left(\bar{P}(\theta) H_{g}(\theta) \bar{P}(\theta)-z\right)^{-1} \quad \text { (III.117) } \\
&\left.-\left(\bar{P}(\theta) H_{0}(\theta) \bar{P}(\theta)-z\right)^{-1}\right] \bar{P}(\theta) W_{g}(\theta) P(\theta) \\
& \operatorname{Rem}_{1}:= P(\theta) W_{g}(\theta) \bar{P}(\theta)\left(\bar{P}(\theta) H_{g} \bar{P}(\theta)-z\right)^{-1} \bar{P}(\theta) W_{g}(\theta) P(\theta) \quad(\text { III.118) } \\
&- g^{2} P(\theta)\left(W_{0,1}(\theta)+W_{1,0}(\theta)\right)\left(\frac{\bar{P}(\theta)}{H_{0}-z}\right)\left(W_{0,1}(\theta)+W_{1,0}(\theta)\right) P(\theta) \\
& \operatorname{Rem}_{2}:=g^{2} P(\theta)\left(W_{0,1}(\theta)+W_{1,0}(\theta)\right)\left(\frac{\bar{P}(\theta)}{H_{0}-z}\right)\left(W_{0,1}(\theta)+W_{1,0}(\theta)\right) P(\theta) \\
&-g^{2} \sum_{\lambda=1}^{2} \int P(\theta) w_{0,1}(\vec{k}, \lambda)\left[\frac{\bar{P}(\theta ; \omega(k))}{H_{0}+e^{-i \vartheta} \omega(k)-z}\right] w_{1,0}(\vec{k}, \lambda) P(\theta) d^{3} k
\end{aligned}
$$

where $P(\theta, \omega):=P_{e l, j}(\theta) \chi_{H_{f}+\omega<\rho_{0}}$,

$$
\operatorname{Rem}_{3}:=P(\theta) W_{g}(\theta) P(\theta)
$$

$$
\begin{gathered}
\operatorname{Rem}_{4}:=g^{2} \sum_{\lambda=1}^{2} \int d k P_{e l, j} w_{0,1}(\vec{k}, \lambda) \\
{\left[\frac{\left(e^{-i \vartheta} H_{f}+E_{j}-z\right) \bar{P}_{e l, j} \chi_{H_{f}<\rho_{0}}}{\left(B_{\theta}(\omega(k))+E_{j}-z\right)\left(H_{e l}-E_{j}+e^{-i \vartheta} \omega(k)\right)}\right] w_{1,0}(\vec{k}, \lambda) P_{e l, j} \chi_{H_{f}<\rho_{0}},}
\end{gathered}
$$

$$
\begin{aligned}
\operatorname{Rem}_{5}:= & g^{2}\left\{\int\left(\sum_{\lambda=1,2} P_{e l, j} w_{0,1}(\vec{k}, \lambda) P_{e l, j} w_{1,0}(\vec{k}, \lambda) P_{e l, j}\right)\right. \\
& {\left.\left[e^{-i \vartheta}\left(H_{f}+\omega(k)\right)+E_{j}-z\right]^{-1} \chi_{H_{f}+\omega(k) \geq \rho_{0}} d k-Z_{j}^{\mathrm{d}}(\theta)\right\} \chi_{H_{f}<\rho_{0}} . }
\end{aligned}
$$


We first rewrite Rem $_{0}$ using the second resolvent equation which yields

$$
\begin{gathered}
\operatorname{Rem}_{0}=P(\theta) W_{g}(\theta) \bar{P}(\theta)\left(\bar{P}(\theta) H_{g}(\theta) \bar{P}(\theta)-z\right)^{-1} \bar{P}(\theta) W_{g}(\theta) \bar{P}(\theta) \\
\left(\bar{P}(\theta) H_{0}(\theta) \bar{P}(\theta)-z\right)^{-1} \bar{P}(\theta) W_{g}(\theta) P(\theta)
\end{gathered}
$$

Then an application of Lemma III.8 gives

$$
\left\|\operatorname{Rem}_{0}\right\| \leq \frac{C g^{3}}{\vartheta^{2} \rho_{0}^{1 / 2}}=\mathcal{O}\left(g^{2+\varepsilon}\right) .
$$

Second, a similar estimate yields

$$
\left\|\operatorname{Rem}_{1}\right\| \leq \frac{C g^{3}}{\vartheta^{2} \rho_{0}^{1 / 2}}=\mathcal{O}\left(g^{2+\varepsilon}\right) .
$$

The derivation of these two estimates, (III.124) and (III.125), is similar to [5, (IV.58)-(IV.62)].

Third, we observe that

$$
\begin{aligned}
& \operatorname{Rem}_{2}=\sum_{\lambda, \lambda^{\prime}=1}^{2} \int d k d k^{\prime}\{ \\
& P(\theta) w_{1,0}(k, \lambda ; \theta) a^{*}(k) a^{*}\left(k^{\prime}\right)\left[\frac{\bar{P}\left(\omega\left(k^{\prime}\right)\right)}{H_{0}+e^{-i \vartheta} \omega\left(k^{\prime}\right)-z}\right] w_{1,0}\left(k^{\prime}, \lambda^{\prime} ; \theta\right) P(\theta) \\
& \quad+P(\theta) w_{0,1}(k, \lambda ; \theta)\left[\frac{\bar{P}(\theta, \omega(k))}{H_{0}+e^{-i \vartheta} \omega(k)-z}\right] w_{0,1}\left(k^{\prime}, \lambda^{\prime} ; \theta\right) a(k) a\left(k^{\prime}\right) P(\theta) \\
& \quad+P(\theta) w_{0,1}(k, \lambda ; \theta) a^{*}\left(k^{\prime}\right) \\
& \quad\left[\frac{\bar{P}\left(\theta, \omega(k)+\omega\left(k^{\prime}\right)\right)}{H_{0}+e^{-i \vartheta}\left(\omega(k)+\omega\left(k^{\prime}\right)\right)-z}\right] w_{1,0}\left(k^{\prime}, \lambda^{\prime} ; \theta\right) a(k) P(\theta) \\
& \left.\quad+P(\theta) w_{1,0}(k, \lambda ; \theta) a^{*}(k)\left[\frac{\bar{P}(\theta)}{H_{0}-z}\right] w_{0,1}\left(k^{\prime}, \lambda^{\prime} ; \theta\right) a\left(k^{\prime}\right) P(\theta)\right\}
\end{aligned}
$$

(compare to $[5,($ IV.66) $])$, which is of the form $P(\theta)\left(\widetilde{W}_{2,0}(\theta)+\widetilde{W}_{0,2}(\theta)+\right.$ $\left.\widetilde{W}_{1,1}(\theta)\right) P(\theta)$. A somewhat lengthy estimate analogous to [5, Lemma IV.9] yields, after using (III.92)-(III.93),

$$
\left\|\operatorname{Rem}_{2}\right\|=\mathcal{O}\left(g^{2+2 \beta(1-\varepsilon)}\right) .
$$

Fourth, we apply (III.94) and directly obtain

$$
\left\|\operatorname{Rem}_{3}\right\|=\mathcal{O}\left(g \rho_{0}^{(1+\beta) / 2}\right)=\mathcal{O}\left(g^{1+(1+\beta)(1-\varepsilon)}\right) .
$$


In order to estimate $\operatorname{Rem}_{4}$, we observe that when restricted to $\operatorname{Ran} \bar{P}_{e l, j}$, the resolvents of $B_{\theta}(\omega(k))+E_{j}-z$ and $H_{e l}-E_{j}+e^{-i \vartheta} \omega(k)$ are bounded by a constant $C \geq 0$. Since, furthermore, $z-E_{j} \in D\left(E_{j}, \rho_{0} / 2\right)$ and $\left\|H_{f} \chi_{H_{f}<\rho_{0}}\right\|=\rho_{0}$, the fraction in the integrand on the right side of (III.121) is bounded in norm by $2 C \rho_{0}$, and we thus obtain

$$
\left\|\operatorname{Rem}_{4}\right\| \leq \mathcal{O}\left(g^{2} \rho_{0}\right) \leq \mathcal{O}\left(g^{4-2 \varepsilon}\right)
$$

Finally, a similar argument, which is along the lines of [5, Lemma IV.12], yields

$$
\left\|\operatorname{Rem}_{5}\right\| \leq \mathcal{O}\left(g^{2} \rho_{0}^{\beta}\right) \leq \mathcal{O}\left(g^{2+2 \beta(1-\varepsilon)}\right) .
$$

Adding up all error terms, taking into account that $0<\beta<1$, we arrive at (III.112).

Lemma III.12. Let $0<\varepsilon<1, \rho_{0}:=g^{2-2 \varepsilon}$ and assume that $\vartheta \in\left(0, \theta_{0}\right)$ and $g>0$ are sufficiently small and such that $\rho_{0}<(\delta / 3) \sin \vartheta$ and $\rho_{0} \geq$ $-2 \pi \vartheta^{-1} \min \left\{\operatorname{Im} \mathcal{A}_{j}\right\}$. Then, for all $z \in \mathcal{A}_{j} \backslash D\left(E_{j}, \rho_{0} / 2\right)$,

$$
\left\|\left(H_{g}(\theta)-z\right)^{-1}\right\| \leq C \operatorname{dist}\left[z, \mathcal{R}_{j}\right]^{-1},
$$

for some constant $C \geq 0$.

Proof: We first consider all eigenvalues $E_{i}$ of $H_{e l}(\theta)$ below $\Sigma-\mu$. Since the eigenvalue closest to $z$ is $E_{j}$, there exists a constant $c>0$ such that

$$
\min _{E_{i} \leq \Sigma-\mu}\left\{\operatorname{dist}\left[z, E_{i}+e^{-\vartheta} \mathbb{R}_{+}\right]\right\} \geq c \vartheta \operatorname{dist}\left[z, E_{j}+e^{-\vartheta} \mathbb{R}_{+}\right]
$$

for any $z \in \mathcal{A}_{j} \backslash D\left(E_{j}, \rho_{0} / 2\right)$. Thus, on $\operatorname{Ran}\left\{P_{\text {disc }}(\theta)\right\}$ (see Eqns. (III.62)(III.62)) we have

$$
\begin{aligned}
\left\|\frac{P_{\text {disc }}(\theta)}{H_{0}(\theta)-z}\right\| & \leq C \max _{E_{i} \leq \Sigma-\mu}\left\{\operatorname{dist}\left[z, E_{i}+e^{-\vartheta} \mathbb{R}_{+}\right]^{-1}\right\} \\
& \leq \frac{C}{c \vartheta} \operatorname{dist}\left[z, E_{j}+e^{-\vartheta} \mathbb{R}_{+}\right]^{-1} .
\end{aligned}
$$

Moreover, since $\rho_{0} \geq-2 \pi \vartheta^{-1} \min \left\{\operatorname{Im} \mathcal{A}_{j}\right\}$, for $g>0$ sufficiently small, we have $-\vartheta / 2 \leq \arg \left(z-E_{j}\right) \leq \pi+\vartheta / 2$, for any $z \in \mathcal{A}_{j} \backslash D\left(E_{j}, \rho_{0} / 2\right)$. Therefore, we also have the estimate

$$
\left\|\frac{B_{\theta}\left(\rho_{0}\right) P_{\text {disc }}(\theta)}{H_{0}(\theta)-z}\right\| \leq C \sup _{r>0} \max _{E_{i} \leq \Sigma-\mu}\left\{\frac{r+\rho_{0}}{\left|E_{i}-z+e^{-i \vartheta} r\right|}\right\} \leq C \vartheta^{-2},
$$


for some $C \geq 0$.

Second, on $\operatorname{Ran}\left\{\bar{P}_{d i s c}(\theta)\right\}$ we employ Lemma III.4 to prove a similar bound,

$$
\left\|\frac{\bar{P}_{d i s c}(\theta)}{H_{0}(\theta)-z}\right\|,\left\|\frac{B_{\theta}\left(\rho_{0}\right) \bar{P}_{d i s c}(\theta)}{H_{0}(\theta)-z}\right\| \leq \frac{C}{\vartheta} \leq \frac{C^{\prime}}{\vartheta} \operatorname{dist}\left[z, E_{j}+e^{-\vartheta} \mathbb{R}_{+}\right]^{-1},
$$

for some constants $C, C^{\prime} \geq 0$. Summarizing (III.133), (III.134), (III.135), and observing that $\operatorname{dist}\left[z, E_{j}+e^{-\vartheta} \mathbb{R}_{+}\right] \geq c \operatorname{dist}\left[z, \mathcal{R}_{j}\right]$, for some $c>0$, we obtain

$$
\left\|\frac{1}{H_{0}(\theta)-z}\right\| \leq \frac{C \vartheta^{-1}}{\operatorname{dist}\left[z, \mathcal{R}_{j}\right]} \quad \text { and }\left\|\frac{B_{\theta}\left(\rho_{0}\right)}{H_{0}(\theta)-z}\right\| \leq C \vartheta^{-1},
$$

for some constant $C \geq 0$ and all $z \in \mathcal{A}_{j} \backslash D\left(E_{j}, \rho_{0} / 2\right)$. So, finally, we obtain the invertibility of $H_{g}(\theta)-z$ and (III.131) from an expansion in a Neumann series as in (III.108)-(III.109).

Acknowledgements: We thank T. Chen and A. Soffer for numerous very helpful discussions on the material presented in this paper and H. Zenk for careful proofreading. We are also grateful to D. Buchholz, F. Hiroshima, F. Klopp, Y. Last, and H. Spohn for valuable comments.

\section{References}

[1] J. Aguilar and J. M. Combes. A class of analytic pertubations for onebody Schrödinger Hamiltonians. Commun. Math. Phys. , 22:269-279, 1971.

[2] S. Albeverio. Scattering theory in a model of quantum fields. I. J. Math. Phys., 14(2):1800-1816, 1972.

[3] S. Albeverio. Scattering theory in a model of quantum fields. II. Helv. Phys. Acta, 45:303-321, 1972.

[4] V. Bach, J. Fröhlich, and I. M. Sigal. Mathematical theory of nonrelativistic matter and radiation. Lett. Math. Phys. , 34:183-201, 1995.

[5] V. Bach, J. Fröhlich, and I. M. Sigal. Quantum electrodynamics of confined non-relativistic particles. Adv. in Math. , 137:299-395, 1998. 
[6] V. Bach, J. Fröhlich, and I. M. Sigal. Renormalization group analysis of spectral problems in quantum field theory. Adv. in Math. , 137:205-298, 1998.

[7] V. Bach, J. Fröhlich, I. M. Sigal, and A. Soffer. Positive commutators and spectrum of nonrelativistic QED. Preprint, 1997.

[8] E. Balslev and J. M. Combes. Spectral properties of Schrödinger operators with dilatation analytic potentials. Commun. Math. Phys. , 22:280294, 1971.

[9] H. Bethe and E. Salpeter. Quantum mechanics of one- and two-electron atoms. In S. Flügge, editor, Handbuch der Physik, XXXV, pages 88-436. Springer, Berlin, 1957.

[10] H. A. Bethe. The electromagnetic shift of energy levels. Phys. Rev. , $72: 339,1947$.

[11] C. Cohen-Tannoudji, J. Dupont-Roc, and G. Grynberg. Photons and Atoms - Introduction to Quantum Electrodynamics. John Wiley, New York, 1991.

[12] C. Cohen-Tannoudji, J. Dupont-Roc, and G. Grynberg. Atom-Photon Interaction. John Wiley, New York, 1992.

[13] H. Cycon, R. Froese, W. Kirsch, and B. Simon. Schrödinger Operators. Springer, Berlin, Heidelberg, New York, 1 edition, 1987.

[14] J. Derezinski and C. Gérard. Asymptotic completeness in quantum field theory. massive Pauli-Fierz Hamiltonians. Preprint, June 1997.

[15] J. Fröhlich and P. Pfeifer. Generalized time-energy uncertainty relations and bounds on lifetimes of resonances. Rev. Mod. Phys., 67:795, 1995.

[16] Ch. Gérard. Asymptotic completeness for the spin-boson model with a particle number cutoff. Rev. Math. Phys., 8:549-589, 1996.

[17] F. Hiroshima. Functional integral representation of a model in QED. Rev. Math. Phys., 9(4):489-530, 1997.

[18] R. Hoegh-Krohn. Asymptotic fields in some models of quantum field theory. III. J. Math. Phys., 11(1):185-189, 1969.

[19] R. Hoegh-Krohn. Boson fields under a general class of cut-off interactions with bounded interaction densities. Commun. Math. Phys., 12:216$225,1969$. 
[20] R. Hoegh-Krohn. Boson fields with bounded interaction densities. Commun. Math. Phys., 17:179-193, 1970.

[21] W. Hunziker. Distortion analyticity and molecular resonance curves. Ann. Inst. H. Poincaré, 45:339-358, 1986.

[22] T. Kato. Perturbation Theory of Linear Operators, volume 132 of Grundlehren der mathematischen Wissenschaften. Springer-Verlag, 2 edition, 1976.

[23] E. H. Lieb. Bound on the maximum negative ionization of atoms and molecules. Phys. Rev. A, 29:3018-3028, 1984.

[24] M. Reed and B. Simon. Methods of Modern Mathematical Physics: Analysis of Operators, volume 4. Academic Press, San Diego, 1 edition, 1978.

[25] M. Reed and B. Simon. Methods of Modern Mathematical Physics: Fourier Analysis and Self-Adjointness, volume 2. Academic Press, San Diego, 2 edition, 1980.

[26] M. Reed and B. Simon. Methods of Modern Mathematical Physics: Functional Analysis, volume 1. Academic Press, San Diego, 2 edition, 1980 .

[27] Mary Beth Ruskai. Absence of discrete spectrum in highly negative ions II. Extension to Fermions. Commun. Math. Phys. , 85:325-327, 1982.

[28] I. M. Sigal. Geometric methods in the quantum many-body problem. nonexistence of very negative ions. Commun. Math. Phys., 85:309-324, 1982.

[29] B. Simon. The definition of molecular resonance curves by the method of exterior complex scaling. Phys. Lett. , A 71:211-214, 1979.

[30] B. Simon. Functional Integration and Quantum Physics. Pure and applied mathematics. Academic Press, New York, 1979.

[31] G. M. Zishlin. Discussion of the spectrum of the Schrödinger operator for systems of many particles. Tr. Mosk. Mat. O. -va, 9:81-120, 1960. 